\title{
Cryptographic Primitives for Information Authentication - State of the Art
}

\author{
Bart Preneel* \\ Katholieke Universiteit Leuven, Dept. Electrical Engineering-ESAT \\ Kardinaal Mercierlaan 94, B-3001 Heverlee, Belgium \\ bart.preneel@esat.kuleuven.ac.be
}

\begin{abstract}
This paper describes the state of the art for cryptographic primitives that are used for protecting the authenticity of information: cryptographic hash functions and digital signature schemes; the first class can be divided into Manipulation Detection Codes (MDCs, also known as one-way and collision resistant hash functions) and Message Authentication Codes (or MACs). The theoretical background is sketched, but most attention is paid to overview the large number of practical constructions for hash functions and to the recent developments in their cryptanalysis. It is also explained to what extent the security of these primitives can be reduced in a provable way to realistic assumptions.
\end{abstract}

\section{Introduction}

In former days, the protection of information was mainly an issue of physical security and selection and motivation of people. To keep information confidential, it was carefully locked in a vault and the discretion of the personnel prevented data from being disclosed. The protection of authenticity relied exclusively on the impossibility of forging documents (as is now still the case e.g., for visa and paper money) and manual signatures. The identification of people relied on eye-to-eye contact and information was transported by couriers.

An important evolution was the communication of information through radio channels, which makes it much more vulnerable to passive eavesdropping. The next step was the processing of information stored on punch cards or tapes with digital computers. The processing capabilities of the computers increased and large amounts of data are now stored on magnetic or optical carriers. Transfer of information is realized through both local and worldwide telecommunication networks. Information processed in computers and under transfer in communication networks is certainly more vulnerable to both passive and active attacks: it is very easy to modify the message or to change sender or recipient. This type of threat is especially important in the context of financial transactions and electronic commerce. Information stored in computers can also easily be modified without leaving any trace. Computers are vulnerable to more specific threats:

* F.W.O. postdoctoral researcher, sponsored by the Fund for Scientific Research Flanders (Belgium).

Appeared in State of the Art and Evolution of Computer Security and Industrial Cryptography, Lecture Notes in Computer Science 1528, Springer-Verlag, pp. 50-105. (C)1998 Springer-Verlag 
computer viruses, Trojan horses, worms, and logic bombs can cause considerable damage. Apart from outsiders breaking into computer systems, the people operating the computer can also be malicious. Copying or changing electronic data is much easier for them than modifying data stored on paper. Another application that will become increasingly important is the protection of the authenticity of pictures and moving images (e.g., video-conferencing). As one can expect that it will become feasible to "edit" moving pictures and make a person say and do things he or she never said or did, it is required that one can guarantee the authenticity of moving images through labeling techniques. This will impose high requirements on the throughput. Other applications where information authentication is important are alarm systems, satellite control systems, distributed control systems, and systems for access control.

The intention of message authentication is to protect the communicating parties against attacks of a third party. However, a different threat emerges when the two communicating parties are mutually distrustful and try to perform a repudiation. This means that sender or receiver will try to modify a message and/or deny to have sent or received a certain message. This is particularly a problem in open environments, such as those envisaged for electronic commerce. A typical example of this fraud can occur in the context of electronic communication of orders to a stockbroker. The customer can place an order to buy shares of company X. If some days later the transaction turns out badly, he will try to deny his order. If on the other hand, the transaction is successful, the stockbroker might claim that he never received the order with the intention to keep the profit. In the case of a dispute, a third party (e.g., a judge) has to take a decision. In paper documents, protection against such a fraud is offered by a handwritten signature and registered mail. It is clear that for electronic messages, a simple name at the end of the message offers no protection at all. This is analogous to the fact that a photocopy of a document with a manual signature is not binding, as one can always produce a bogus document with cut and paste operations. Similarly, a manual signature under a message sent through a telefax or via a computer network has no value.

This paper discusses cryptographic techniques that can provide solutions to the problems discussed above. In the case of protection against a third party, the term symmetric authentication is used, and the cryptographic techniques employed are cryptographic hash functions. In the case of protection against repudiation, one uses the term asymmetric authentication and the corresponding cryptographic techniques are digital signatures.

In a first part of this paper, the difference between symmetric authentication algorithms and digital signatures is explained in more detail, and the need for both techniques is demonstrated. Three approaches to solve cryptographic problems are compared. The principles of unconditionally secure authentication are explained, and the complexity theoretic approach to cryptography is discussed. The second part deals with cryptographic hash functions; MDCs and MACs are treated separately. First definitions are given, and subsequently applications are discussed. Then an extensive overview is given of proposed schemes together

Appeared in State of the Art and Evolution of Computer Security and Industrial Cryptography, Lecture Notes in Computer Science 1528, Springer-Verlag, pp. 50-105. (C)1998 Springer-Verlag 
with a taxonomy for methods of attack. In a third part a brief overview is given of practical digital signature schemes.

\section{Information Authentication}

An important distinction in authentication is whether one considers symmetric authentication, between mutually trusting parties, or asymmetric authentication, where one party protects a document, and the second party can only verify a document. The next sections discuss the two approaches.

\subsection{Symmetric Authentication}

The protection of the authenticity of information includes two aspects:

- the protection of the originator of the information, or in ISO 7498-2 terminology [85] data origin authentication.

- the fact that the information has not been modified, or in ISO 7498-2 terminology [85] the integrity of the information.

The first aspect can be present in a hidden way if one implicitly trusts the source of the information (e.g., information that is read from the hard disk of a private laptop). Other aspects that can be important are the timeliness, the sequence with respect to other messages, and the intended recipient(s).

Until the late seventies, it was generally believed that encryption of information suffices to protect its authenticity. The argument was that if a ciphertext resulted after decryption in meaningful information, it should be originated with someone who knows the secret key, guaranteeing authenticity of message and sender. Two counterexamples will show that this belief is wrong: the protection of integrity is dependent on the encryption algorithm and on the mode in which the algorithm is used.

The Vernam cipher [170] adds a random key string modulo 2 to the plaintext. It was shown by Shannon [160] that this offers perfect secrecy: an eavesdropper learns no additional information on the plaintext from observing the ciphertext, no matter how much computational power she has. However, an active attacker can change any bit of the plaintext by simply flipping the corresponding bit of the ciphertext. This observation is also valid for any additive stream cipher and for the Output FeedBack (OFB) mode of any block cipher. It holds partially if the cipher is used in Cipher FeedBack (CFB) or Cipher Block Chaining (CBC) mode; for a definition of these modes, see the FIPS or ISO/IEC standards [62, 89].

If a plaintext longer than one block is enciphered with a block cipher in ECB mode, an active attacker can easily reorder the blocks. Due to the selfsynchronization property of the Cipher Feedback Mode (CFB), any modification in the ciphertext will cause a corresponding modification to the plaintext and will subsequently garble the next part of the plaintext. When the error has shifted out of the feedback register, the ciphertext will be deciphered correctly again. If

Appeared in State of the Art and Evolution of Computer Security and Industrial Cryptography, Lecture Notes in Computer Science 1528, Springer-Verlag, pp. 50-105. (C)1998 Springer-Verlag 
the last part of the ciphertext is modified, it is completely impossible to detect this. If the garbling occurs in the middle of the plaintext, it can only be detected based on redundancy, as will be discussed below.

In other modes (like the CBC mode) every ciphertext bit is a complex function of the previous plaintext bits and an initial value. If the modification of a single ciphertext bit results in $t$ bits of the plaintext being garbled, the probability that the new plaintext will be accepted as meaningful equals $2^{-t D}$, where $D$ is the redundancy in the information. In the case of natural language, coded with 5 bits per character, the redundancy per bit $D \approx 0.74$, and this probability is equal to $2^{-47.4}$ for $t=64$. However, if $D=0$ all messages are meaningful, and encryption offers no authentication, independently of the encryption algorithm or of the mode. Note that even if redundancy is present, a human checker or a designated computer program is required to check its presence. If an intelligent attacker comes into play, the natural redundancy offers little protection, with the exception of a ciphertext only setting (in that case an attacker does not know beforehand what the corresponding plaintext will be; but there exist applications for which such a 'weak' attack may cause serious problems). If however a number of plaintext-ciphertext pairs are known, the encryption becomes vulnerable to splicing attacks. In a chosen plaintext or chosen ciphertext setting, none of the standard encryption modes offers any protection against such attacks.

In order to protect the integrity, a special form of redundancy has to be added, and if the information is to be linked with an originator, a secret key has to be involved in the process (this assumes a coupling between the person and his key), or a separate integrity channel has to be provided. Hence two basic methods can be identified.

- The first approach is analogous to that of a symmetric cipher, where the secrecy of large data quantities is based on the secrecy and authenticity of a short key. In this case the authentication of the information also relies on the secrecy and authenticity of a key. To achieve this goal, the information is compressed to a quantity of fixed length, which is called a hash result. Subsequently the hash result is appended to the information. The function that performs this compression operation is called a hash function. The basic idea of the protection of the integrity is to add redundancy to the information. The presence of this redundancy allows the receiver to make the distinction between authentic information and bogus information.

In order to guarantee the origin of the data, a secret key that can be associated to the origin intervenes in the process. The secret key can be involved in the compression process or can be used to protect the hash result and/or the information. In the first case the hash result is called a Message Authentication Code or MAC, while in the latter case the hash result is called a Manipulation Detection Code or MDC.

- The second approach consists of basing the authenticity (both integrity and origin authentication) of the information on the authenticity of a Manipulation Detection Code or MDC. A typical example for this approach is a computer user who will calculate an MDC for all its important files. He can

Appeared in State of the Art and Evolution of Computer Security and Industrial Cryptography, Lecture Notes in Computer Science 1528, Springer-Verlag, pp. 50-105. (C)1998 Springer-Verlag 
store this collection of MDCs on a floppy, that is locked in his safe, or he can write them down on a piece of paper. If he has to transfer the files to a remote friend, he can simply send the files and communicate the MDCs via telephone. The authenticity of the telephone channel is offered here by voice identification.

Note that the addition of redundancy is not sufficient. Additional measures are required to protect against high level attacks, such as the replay of an authenticated message (cf. Sect. 8.3).

Both approaches do not work if sender and receiver do not trust each other. In the first approach, they share the same secret key. If one of the parties claims that the information was changed by the other party, a judge cannot make a distinction between them, even if they disclose their common secret key. The second approach can only provide non-repudiation if both parties trust the authenticity of the MDC: in practice however this is difficult to realize, as both parties have a similar access to that channel.

\section{$2.2 \quad$ Asymmetric Authentication}

If one wants to be protected against insider attacks, one needs an electronic equivalent of a manual signature. In this case a third party will be able to distinguish between both parties, based on the fact that the capabilities of both parties are different. The concept of a digital signature was introduced by Diffie and Hellman in 1976 [50]. The requirements are that the signature depends on the information to be signed (since it is not physically connected to the document) and that the signer is the only person who can produce a signature (this means no one else can forge a signature, which implies that the signer cannot deny that he has produced it). A digital signature scheme consists of the following elements:

- an initialization phase (e.g., key generation and general setup);

- a signing process, where the signature is produced;

- a verification process, where the receiver (or a judge) verifies whether the signature is correct.

Digital signatures in this sense can be produced based on tamper resistant devices, conventional one-way functions, or public-key techniques. Sect. 9 provides a brief overview of practical signature schemes.

Several generalizations have been defined, with more players in the game and with different notions of security. Examples of such extensions are: arbitrated signatures, where the signing and verification process involves interaction with a third party, group signatures, where signers and/or verifies are members of a group, blind signatures, where the signer signs a 'blinded' or 'masked' message, and undeniable signatures, where the signature can only be verified with the cooperation of the signer. For a more extensive treatment of digital signature schemes the reader is referred to the Handbook of Applied Cryptography [115] and the work by Pfitzmann [133].

Appeared in State of the Art and Evolution of Computer Security and Industrial Cryptography, Lecture Notes in Computer Science 1528, Springer-Verlag, pp. 50-105. (C)1998 Springer-Verlag 


\section{Three Approaches in Cryptography}

In modern cryptography, three approaches can be identified; they differ in the assumptions about the capabilities of an opponent, in the definition of a cryptanalytic success, and in the notion of security. Our taxonomy deviates from the approach by Simmons [162], and is based on the taxonomy for stream ciphers of Rueppel [157]. A first method is based on information theory, and it offers unconditional security, i.e., security independent of the computing power of an adversary. The complexity theoretic approach starts from an abstract model for computation, and assumes that the opponent has limited computing power. The system based approach tries to produce practical solutions, and the security estimates are based on the best algorithm known to break the system and on realistic estimates of the necessary computing power or dedicated hardware to carry out the algorithm. Simmons lumps the second and third approach together as computationally secure [162], and Rueppel defines a fourth approach in which the opponent has to solve a problem with a large size (namely examining a huge publicly accessible random string) [157]; it can be considered as both computationally secure and information theoretically secure.

\subsection{Information Theoretic Approach}

This approach results in unconditionally secure solutions, which implies that the security of the system is independent of the computing power of the opponent. E.g., for privacy protection, it has been shown by Shannon [160] that unconditional privacy protection requires that the entropy of the key is lower bounded by the entropy of the plaintext. Note that both unconditional privacy and unconditional authenticity are only probabilistic: even if the system is optimal with respect to some definition, the opponent has always a non-zero probability to cheat. However, this probability can be made exponentially small. Until recently, it was widely believed that unconditionally secure schemes for authentication were as impractical as the Vernam scheme; however, during the last years schemes have been developed which are extremely efficient both in terms of key usage and in terms of computation.

The idea of unconditionally secure authentication dates back to the early seventies, when Simmons was developing for Sandia National Laboratories a system for the verification of treaty compliance, such as the comprehensive nuclear testban treaty between the USA and the USSR [163]. The first technical solution to the problem appeared in 1974 in a paper by Gilbert et al. [72]. Subsequently the theory has been developed further by Simmons, analogous to the theory of secrecy systems that was invented by Shannon [160]. An overview of this theory can be found in the work of Simmons [162] and Stinson [165,167]. Recent developments can be found in the work of Kabatianskii et al. [97].

From the brief but clear summary by Massey in [111] we can cite the following statement "The theory of authenticity is in many ways more subtle than the corresponding theory of secrecy. In particular, it is not at all obvious how "perfect authenticity" should be defined." This is caused by the fact that there

Appeared in State of the Art and Evolution of Computer Security and Industrial Cryptography, Lecture Notes in Computer Science 1528, Springer-Verlag, pp. 50-105. (c)1998 Springer-Verlag 
are different bounds that can be met with equality. In this section we will give the basic definitions, discuss briefly a taxonomy, and give (without proof) the most important theoretical bounds. We conclude with a brief overview of the recent efficient construction.

Definitions and Notation. In the literature on this subject, the information to be communicated is called the source state, while the information that will be sent to the receiver is called the message. A mapping between the space of source states and the message space is called an encoding rule. The set of source states, messages, and encoding rules is called an authentication code. In the following we will keep the more usual terminology of plaintext, ciphertext, and key. The set of plaintext, ciphertexts, and keys will be denoted with $\{P\},\{C\}$, and $\{K\}$ respectively. The size of plaintext, ciphertext, and key space will be denoted with $p, c$, and $k$.

As usually in cryptography, the main players are the sender Alice, who wants to send some information to the receiver Bob; the opponent of Alice and Bob is the active eavesdropper Eve, who can perform three types of attacks:

- Eve can send a fraudulent cryptogram to Bob as if it came from Alice (impersonation attack);

- Eve can wait until she observes a cryptogram and replace it by a different cryptogram (substitution attack);

- Eve can choose freely between both strategies (deception attack).

The probability of success (when the strategy of Eve is optimal) will be denoted with $P_{i}, P_{s}$, and $P_{d}$ respectively. A first result that follows from Kerckhoffs' assumption $^{1}$ (namely that the strategy to choose the key is known by Eve) is that

$$
P_{d}=\max \left(P_{i}, P_{s}\right) \text {. }
$$

A basic distinction that can be made in this theory is between authentication codes with and without secrecy. In the latter case the plaintext can be derived easily from the ciphertext. The corresponding codes are called Cartesian or systematic. In this case one can write the ciphertext as the concatenation of the plaintext $P$ with an authenticator, Message Authentication Code, or MAC. The number of authenticators is denoted with $\rho$. In the following the assumption will be made that the authenticator is a deterministic function of the plaintext and the key (in technical terms: only authentication codes without splitting are considered).

Bounds on Authentication Codes. The simplest bound that can be given is for an impersonation attack: if Eve selects $C$ completely at random from the

\footnotetext{
${ }^{1}$ The Dutchman A. Kerckhoffs (1853-1903) was the first to state the principle that
} the security of a cipher must reside entirely in the secrecy of its key.

Appeared in State of the Art and Evolution of Computer Security and Industrial Cryptography, Lecture Notes in Computer Science 1528, Springer-Verlag, pp. 50-105. (C)1998 Springer-Verlag 
$c$ cryptograms that occur with nonzero probability in the authentication code, her probability of success can be lower bounded by the following expression:

$$
P_{i} \geq \frac{p}{c}
$$

which is called the combinatorial bound for impersonation. For Cartesian codes this bound becomes $P_{i}>1 / \rho$.

If no splitting occurs, a similar bound can be given for substitution:

$$
P_{s} \geq \frac{p-1}{c-1}
$$

which is called the combinatorial bound for substitution.

The next bound is based on the concept of mutual information, and is also called the 'authentication channel capacity' theorem:

$$
P_{d} \geq 2^{-I(C ; K)} \text {. }
$$

For the shortest proof known until now and a discussion of the recent improvements on this bound by Johannesson and Sgarro the reader is referred to [111]. A formulation in words is that the difference between the amount of information transmitted through the channel and that needed by the receiver to resolve his uncertainty about the plaintext can be used to authenticate the plaintext, and conversely no better result can be achieved. One can now show that

$$
P_{d} \geq \frac{1}{\sqrt{k}} .
$$

This means in practice that the security level $\left(-\log _{2} P_{d}\right)$ is at most half the key size in bits. For $l$-fold secure schemes where an opponent can first observe $l$ ciphertexts, this has been extended by Fåk [59] to

$$
P_{d} \geq \frac{1}{\sqrt[1+1]{k}}
$$

The next step is to define perfect authenticity to mean that equality holds in the equation for the authentication channel capacity. Note that even if a system is perfect, $P_{d}$ will only be small if the cryptogram $C$ reveals much information about the key $K$. Two simple examples of perfect authentication codes are given in Table 1.

Massey shows that perfect authenticity schemes are less efficient in terms of key bits than schemes that provide perfect secrecy [110]. To be more in line with the rest of this paper, from now on $p$ and $c$ will be powers of two: an $r$-bit message will be authenticated with a $m$-bit MAC, or $p=2^{r}$ and $c=2^{r+m}$. [110]: if an $r$-bit plaintext is mapped to an $(r+m)$-bit ciphertext, the number of key bits per plaintext bit for perfect authenticity with $P_{i}$ and $P_{s}$ equal to the combinatorial bound is at least $m / r$, which is much larger than 1 if $r$ is small. Note that $P_{i}=1 / 2^{m}$, so $m$ cannot be small. However, unlike for secrecy, authenticity does not have any need for perfect schemes. As will be explained below, if $P_{s}$ is allowed to be slightly smaller than $P_{i}$, the key size can be close to minimal (that is, as indicated by (1)), and this for both short and very long messages.

Appeared in State of the Art and Evolution of Computer Security and Industrial Cryptography, Lecture Notes in Computer Science 1528, Springer-Verlag, pp. 50-105. (c)1998 Springer-Verlag 
Table 1. A perfect Cartesian authentication code and a perfect authentication code that also provides perfect secrecy [111]

\begin{tabular}{|c|c|c|}
\hline$K$ & $P=0$ & $P=1$ \\
\hline 00 & 00 & 10 \\
01 & 01 & 11 \\
10 & 00 & 11 \\
11 & 01 & 10 \\
\hline
\end{tabular}

\begin{tabular}{|c|c|c|}
\hline$K$ & $P=0$ & $P=1$ \\
\hline 00 & 00 & 10 \\
01 & 01 & 00 \\
10 & 11 & 01 \\
11 & 10 & 11 \\
\hline
\end{tabular}

Practical Cartesian Authentication Codes. This section presents more practical authentication schemes. The problem with the perfect constructions is that a key of at least $2 m$ bits is required. Moreover, a theorem by Stinson [166], states that if $P_{i}=P_{s}=1 / 2^{m}$, the number of plaintexts is at most a linear function of the number of keys. The idea is to reduce the key size at the cost of a small increase of $P_{s}$. Such constructions were already published by Carter and Wegman in the late seventies under the name of strongly universal hash functions [28, 171]. Kabatianskii et al. [97] showed that if $P_{s}$ exceeds $P_{i}$ by an arbitrarily small amount, the number of plaintexts grows exponentially with the number of keys. This research developed from exploring connections to the rich theory of error-correcting codes. The price to be paid is that $m$ has to be slightly larger to achieve the same value of $P_{d}$ as for the perfect schemes.

Krawczyk has considered universal hash functions that are linear with respect to bitwise exor $[104,105]$. This property makes it easier to reuse the authentication code (with the same key): one encrypts the $m$-bit hash result for each new message using a one-time pad. This approach leads to very simple constructions and proofs for constructions based on polynomials and Linear Feedback Shift Registers (LFSRs). For the case $P_{s}=2 \cdot P_{i}$, the most efficient construction to date is that of Kabatianskii et al. [97]. All these constructions use a composition: the bulk of the hashing is done with a function with weaker properties, and the stronger properties (which require a larger keys size) are only used for the final stage. Several of these schemes are based on the evaluation of polynomials over a finite field; Shoup [161] and Afanassiev et al. [1] have studied efficient software implementations of this primitive. These schemes offer the best performance for small key sizes and hash results.

A second line of research has been to improve the speed at the cost of an increased key size and size of the hash result. Rogaway has introduced bucket hashing in [154]; a slower variant with shorter keys was introduced by Johansson in [93]. Halevi and Krawczyk have designed an extremely fast scheme which makes optimal used of the multiply and accumulate instruction of the Pentium MMX processor (1 Gbit/s on a $200 \mathrm{MHz}$ Pentium Pro) [81]. Such high speeds can be obtained because authentication codes have combinatorial rather than cryptographic properties.

As an example, a construction based on Reed-Solomon codes is described; this scheme was first published by Mehlhorn and Vishkin [113]. The key consists

Appeared in State of the Art and Evolution of Computer Security and Industrial Cryptography, Lecture Notes in Computer Science 1528, Springer-Verlag, pp. 50-105. (C)1998 Springer-Verlag 
of 2 elements of $G F\left(2^{m}\right)$ denoted with $\mu$ and $\nu$. The argument $x$ is split into elements of $G F\left(2^{m}\right)$ denoted with $X_{1}, X_{2}, \ldots, X_{t}$, hence $r=t \cdot m$. The function is then defined as follows:

$$
g(x)=\mu+\sum_{i=1}^{t} X_{i} \cdot \nu^{i},
$$

where the addition and the multiplication are in $G F\left(2^{m}\right)$. It can be shown that this yields an authentication code with $P_{i}=2^{-m}$ and $P_{s}=(r / m) / 2^{m}=t / 2^{m}$. It is clear that this scheme can be generalized to any finite field. If $r=m$ this construction reduces to the construction for a perfect authentication code by Gilbert et al. [72].

The schemes discussed in this section have the property that the secret key can be used for one message only. However, if the construction is additive, it is sufficient to refresh the last part of the key, which 'encrypts' the output (the value of $\mu$ in the last example). In practice, this value can be computed from a short key by using a pseudo-random bit generator (with only computational or heuristic security), as suggested by Brassard [25]; as a consequence, the resulting scheme is no longer unconditionally secure, but it is clear on what the security is based. It is then essential that this pseudo-random bit generator is very strong. For long messages, the overhead of computing the key is compensated by the much higher evaluation speed of the authentication code. For short messages, conventional MAC algorithms as discussed in Sect. 4.3 are still more efficient.

Unconditionally Secure Digital Signatures. Under certain assumptions, it is possible to construct digital signatures that are unconditionally secure for signer or receiver (this implies that even infinite computing power will not enable the other party to cheat with a significant probability). If the number of participants is limited, the construction of Chaum and Roijakkers [32] provides unconditionally secure signature schemes, that is, digital signatures that are unconditionally secure for both signer and receiver.

\subsection{Complexity Theoretic Approach}

The approach taken here is to define a model of computation, like a Turing machine or a Boolean circuit [3]. All computations in this model are parameterized by a security parameter, and only algorithms or circuits that require asymptotically polynomial time and space in terms of the size of the input are considered feasible. The next step is then to design cryptographic systems that are provably secure with respect to this model. This research program has been initiated in 1982 by Yao [172] and tries to base cryptographic primitives on general assumptions. Examples of such primitives are: secure message sending, cryptographically secure pseudo-random generation, digital signatures, and bit commitment. Examples of general assumptions to which these primitives can be reduced are the existence of one-way functions, injections, or permutations, and

Appeared in State of the Art and Evolution of Computer Security and Industrial Cryptography, Lecture Notes in Computer Science 1528, Springer-Verlag, pp. 50-105. (C)1998 Springer-Verlag 
the existence of trapdoor one-way permutations. A third aspect is the efficiency of the reduction, i.e., the number of executions of the basic function to achieve a cryptographic primitive, and the number of interactions between the players in the protocol. For practical applications, the tightness of the redundancy is also important, i.e., the relation between the security of the building blocks and that of the resulting scheme.

An important research goal is to reduce cryptographic primitives to weaker assumptions, with as final goal to prove that the reduction is optimal. One can also try to improve the efficiency of a reduction, possibly at the cost of a stronger assumption. If someone wants to build a concrete implementation, he will have to choose a particular one-way function, permutation, etc. The properties of a particular problem that is believed to be hard can be used to increase the efficiency of the solutions. Examples of problems that have been intensively used are the factoring of a product of two large primes, the discrete logarithm problem modulo a prime and modulo a composite that is the product of two large primes, and the quadratic residuosity problem (see for example Sect. 5.3).

The complexity theoretic approach has several advantages:

1. It results in provable secure systems, based on a number of assumptions.

2. The constructions of such proofs requires formal definitions of the cryptographic primitives and of the security of a cryptographic primitive.

3. The assumptions on which the security of the systems is based are also defined formally.

The disadvantage is that the complexity theoretic approach has only a limited impact on practical implementations, due to limitations that are inherently present in the models.

1. In complexity theory, a number of operations that is polynomial in the size of the input is considered to be feasible, while a superpolynomial or exponential number of operations in the size of the input is infeasible. In an asymptotic setting, abstraction is made from both constant factors and the degrees of the polynomials. This implies that this approach does not always provide information on the security of concrete instances (a practical problem has a finite size). Moreover, the scheme might be impractical because the number of operations to be carried out is polynomial in the size of the input but impractically large. Even if the cost in efficiency of a provable scheme compared to an ad hoc scheme is moderate, one is not always inclined to select provable secure solutions. Recently more attention has been paid to this aspect, and several results are known which provide tight and efficient reductions to the security of primitives of fixed size. Example are the security analysis of HMAC and CBC-MAC by Bellare et al. [10,14].

2. The complexity theoretic approach yields only results on the worst case or average case problems in a general class of problems. However, cryptographers studying the security of a scheme are more interested in the subset of problems that is easy.

3. Complexity usually deals with single isolated instances of a problem. A cryptanalyst often has a large collection of statistically related problems to solve.

Appeared in State of the Art and Evolution of Computer Security and Industrial Cryptography, Lecture Notes in Computer Science 1528, Springer-Verlag, pp. 50-105. (C)1998 Springer-Verlag 
A few theoretical results on information authentication will be summarized briefly. Their importance will only be appreciated after reading Sect. 4. Naor and Yung have introduced the concept of a Universal One-Way Hash Function (UOWHF) [128] (not to be confused with a universal hash function, cf. Sect. 3.1). The idea behind a UOWHF is that first the input is selected and subsequently (and independently) the hash function. In this case it does not help an opponent to find collisions for the hash function (cf. Sect. 4.2). Naor and Yung show how to use a UOWHF to build a signature scheme. An important result is that it is sufficient to have a UOWHF that compresses a single bit to construct a UOWHF that compresses an arbitrary number of bits. Several authors have subsequently improved their construction. A key result by Rompel [156] is a (very inefficient) construction for a UOWHF based on any one-way function, which is the weakest possible assumption. Bellare and Rogaway propose efficient constructions for UOWHFs, which they call 'target collision resistant hash functions' or TCRs [17]. They also show that if such a primitive is built from a compression function (cf. Sect. 5.1), a different approach is required than for the hash functions discussed in Sect. 4.

A second result by Damgård [40,41] is a construction for a CRHF (Collision Resistant Hash Function) based on a collision resistant compression function with fixed size input (see Theorem 2 in Sect. 5.1).

A recent result by Simon [164] provides a motivation to treat collision resistant hash functions as independent cryptographic primitives. He shows hat no provable construction of a CRHF can exists based on a "black box" one-way permutation, i.e., a one-way permutation treated as an oracle.

\subsection{System Based or Practical Approach}

In this approach schemes with fixed dimensions are designed and studied, paying special attention to the efficiency of software and hardware implementations. The goal of this approach is to ensure that breaking a cryptosystem is a difficult problem for the cryptanalyst.

By trial and error procedures, several cryptanalytic principles have emerged, and the designer intends to avoid attacks based on these principles. Typical examples are statistical attacks and meet-in-the-middle attacks. An overview of these principles for MDCs and MACs is presented in Sect. 6 and 8 .

The second aspect is to design building blocks with provable properties. These building blocks are not only useful for cryptographic hash functions, but also for the design of block ciphers and stream ciphers. Typical examples are statistical criteria, diffusion and confusion, correlation, and non-linearity criteria. For more details, see Preneel et al. [138].

Third, the assembly of basic building blocks to design a cryptographic hash functions can be based on theorems. Results of this type are often formulated and proven in a complexity theoretic setting, but can easily be adapted to a more practical definition of "hardness" that is useful in a system based approach. A typical example is the theorem discovered independently in [42] and [118], stating

Appeared in State of the Art and Evolution of Computer Security and Industrial Cryptography, Lecture Notes in Computer Science 1528, Springer-Verlag, pp. 50-105. (C)1998 Springer-Verlag 
that a collision-resistant hash function can always be constructed if a collisionresistant function exists, where the first reference uses a complexity theoretic approach and the second a more practical definition.

\section{Cryptographic Hash Functions}

Sect. 2 has explained how the authenticity of information can be verified through the protection of the secrecy and/or the authenticity of a short imprint or hash result. This section presents definitions and applications of hash functions that use a secret key (Message Authentication Codes or MACs) and for hash functions that do not make use of a secret key (Manipulation Detection Codes or MDCs).

A brief discussion of the existing terminology can clarify the confusion in the literature. The term hash functions originates historically from computer science, where it denotes a function that compresses a string of arbitrary length to a string of fixed length. Hash functions are used to allocate as uniformly as possible storage for the records of a file. The name "hash functions" has also been widely adopted for cryptographic hash functions or cryptographically strong compression functions, but the result of the hash function has been given a wide variety of names in cryptographic literature: hash code, hash total, hash result, imprint, (cryptographic) checksum, compression, compressed encoding, seal, authenticator, authentication tag, fingerprint, test key, condensation, Message Integrity Code (MIC), message digest, etc. The terms MAC and MDC originated from US standards and are certainly not perfect (a MAC or an MDC are actually no codes, and both can serve for message authentication), but the adoption of these terms offers a pragmatic solution. One example of the confusion is that "checksum" is associated with the well known Cyclic Redundancy Checks (CRC) that are of no use for cryptographic applications. In this paper the names MAC and MDC will also be used for the hash result obtained with a $\mathrm{MAC}$ and an MDC respectively; the algorithm that computes the MAC result is often called the MAC algorithm. Sometimes a MAC algorithm is called a keyed hash function, but then one has to use for an MDC the artificial term unkeyed or keyless hash function. Moreover, some people understand by this definition an MDC that has an input containing a secret key. The class of MDCs will be further divided into one-way hash functions (OWHF) and collision resistant hash functions (CRHF). The term collision resistant hash function (CRHF) is preferable over strong one-way hash function, as it explains more clearly the actual property that is satisfied. The term collision free hash function proposed by Damgård is also more explicit, but can be slightly misleading: in fact collisions do exist, but it should be hard to find them. An alternative that was proposed by Zheng et al. in [175] is collision intractible hash functions. The term weak one-way hash function was proposed by Merkle in [118], in order to stress the difference with a strong or collision resistant hash function. Finally note that in a complexity theoretic context the concept of universal one-way hash function

Appeared in State of the Art and Evolution of Computer Security and Industrial Cryptography, Lecture Notes in Computer Science 1528, Springer-Verlag, pp. 50-105. (C)1998 Springer-Verlag 
(UOWHF) was defined by Naor and Yung in [128] (cf. Sect. 3.2). The relation between several cryptographic hash functions has been summarized in Fig. 1.

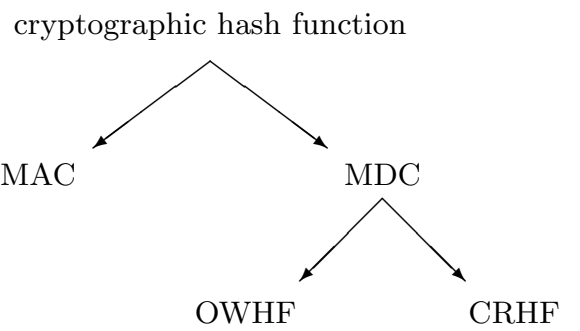

Fig. 1. A taxonomy for cryptographic hash functions

In the following the hash function will be denoted with $h$, and its argument, i.e., the information to be protected with $X$. The image of $X$ under the hash function $h$ will be denoted with $h(X)$; the notation $h_{K}(X)$ will be used for a MAC, with $K$ the secret key. It will be assumed that the description of the hash function $h$ is publicly known; the computation of an MDC does not require any secret information, while the only secret information for a MAC algorithm lies in the key. This is an extension of Kerckhoffs' principle. A second assumption is that given the inputs, the computation of the hash result must be "easy".

\subsection{One-Way Hash Function (OWHF)}

The concept of one-way hash functions was introduced by Diffie and Hellman in [50]. The first informal definition was apparently given by Merkle $[116,118]$ and Rabin [146].

Definition 1 A one-way hash function is a function $h$ satisfying the following conditions:

1. The argument $X$ can be of arbitrary length and the result $h(X)$ has a fixed length of $n$ bits (with $n \geq 64 \ldots 80$ ).

2. The hash function must be one-way in the sense that given a $Y$ in the image of $h$, it is "hard" to find a message $X$ such that $h(X)=Y$ (preimage resistant) and given $X$ and $h(X)$ it is "hard" to find a message $X^{\prime} \neq X$ such that $h\left(X^{\prime}\right)=h(X)$ (second preimage resistant).

Note that this last condition (finding a second preimage is "hard") differs from the intuitive concept of one-wayness, namely that it is "hard" to find a preimage $X$ given only $h$ and the value of $h(X)$. It is clear that for permutations or injective functions only preimage resistance is relevant. The relation between preimage resistance and second preimage resistance is discussed in $[115,134]$. Formal definitions of a OWHF can be obtained through insertion of a formal definition of "hard" and "easy" in combination with the introduction of a security parameter.

Appeared in State of the Art and Evolution of Computer Security and Industrial Cryptography, Lecture Notes in Computer Science 1528, Springer-Verlag, pp. 50-105. (C)1998 Springer-Verlag 


\subsection{Collision Resistant Hash Function (CRHF)}

The importance of collision resistance for hash functions used in digital signature schemes was first pointed out by Yuval in [173]. The first formal definition of a CRHF was apparently given by Damgård [40,41]. An informal definition was given by Merkle in [118].

Definition $2 A$ collision resistant hash function is a function $h$ satisfying the following conditions:

1. The argument $X$ can be of arbitrary length and the result $h(X)$ has a fixed length of $n$ bits (with $n \geq 128 \ldots 160$ ).

2. The hash function must be one-way, i.e., preimage resistance and second preimage resistant.

3. The hash function must be collision resistant: this means that it is "hard" to find two distinct messages that hash to the same result.

Finding a second preimage cannot be easier than finding a collision: therefore the second preimage condition in this definition is redundant. However, preimage resistance is not necessarily implied by collision resistance, while it is required for certain applications. Damgård provides some definitions and conditions under which collision resistance implies preimage resistance [42]; see also Gibson's comment in [70].

For a practical definition, several options are available. In the case of "ideal security," defined by Lai and Massey [107], producing a (second) preimage requires about $2^{n}$ operations and producing a collision requires about $2^{n / 2}$ operations (see Sect. 6), but in practice it may be sufficient that both are computationally infeasible.

\subsection{Message Authentication Code (MAC)}

Message Authentication Codes have been used for a long time in the banking community and are thus older than the open research in cryptology that started in the mid seventies. However, MAC algorithms with good cryptographic properties were only introduced after the start of open research in the field. The first reference to a MAC is a 1972 patent application by Simmons et al. (reference 10. in [163]).

Definition 3 A MAC is a function $h$ satisfying the following conditions:

1. The argument $X$ can be of arbitrary length and the result $h_{K}(X)$ has a fixed length of $n$ bits (with $n \geq 32 \ldots 64$, cf. Sect. 8).

2. Given $h$ and $X$, it is "hard" to forge a MAC on a new message, that is, to determine $h_{K}(X)$ with a probability of success "significantly higher" than $1 / 2^{n}$. Even when many pairs $\left\{X_{i}, h_{K}\left(X_{i}\right)\right\}$ are known, where the $X_{i}$ have been selected (sequentially) by the opponent, it is "hard" to compute $h_{K}\left(X^{\prime}\right)$ for any $X^{\prime} \neq X_{i}$.

Appeared in State of the Art and Evolution of Computer Security and Industrial Cryptography, Lecture Notes in Computer Science 1528, Springer-Verlag, pp. 50-105. (C)1998 Springer-Verlag 
The last attack is called an adaptive chosen text attack; see Sect. 8 for more details. One distinguishes between forgery attacks and key recovery attacks: a forgery allows to determine the MAC on a new message; a key recovery attack is much more serious as it allows to forge any arbitrary message. Note that resistance to forgery attacks implies that the MAC algorithm should be both one-way and collision resistant for someone who does not know the secret key $K$. This definition leaves the problem open whether or not a MAC algorithm should be one-way or collision resistant for someone who knows $K$. An example where this property could be useful is the authentication of multi-destination messages [123].

\subsection{Applications of Cryptographic Hash Functions}

Cryptographic hash functions can be used to provide for information authentication with and without secrecy. It will also be shown that hash functions play a very important role in the design of digital signature schemes. Other applications that will not be discussed here are the protection of pass-phrases (probably the first application of one-way functions), the commitment to a string without revealing it [43], key derivation, and the construction of block ciphers.

Authentication Without Secrecy. In this case there is only a plaintext available, which significantly reduces the number of options.

$M A C$. In order to protect the authenticity of the information, one computes the MAC and appends it to the information. The authenticity of the information now depends on the secrecy and authenticity of the secret key and can be protected and verified by anyone who is privy to this key. The protection of authenticity has thus been reduced to the problem of securely establishing shared keys. This approach can against outsider attacks, as all parties involved have the same capabilities and hence should trust each other.

The scheme can be made even more secure (but less practical) if also the authenticity and/or secrecy of the MAC of every plaintext is protected. A possible implementation could consist of an exchange of messages over a high speed communication link, while the corresponding MACs are sent over a slower channel, which protects authenticity and/or secrecy. For a strong MAC algorithm, this additional protection is not necessary.

$M D C$. If an MDC is used, authenticity of the information is transferred to the authenticity of a string of fixed length. The advantage over a MAC is that there is no need for management of secret keys. In exchange for this, an authentic channel has to be provided for every MDC. This means that the capacity of the channel will increase with the number of messages. Although the lifetime of a key is also related to the number of times it has been used, it is clear that the authentic channel for the MDC will need a significantly greater capacity than the channel that protects both authenticity and secrecy of the key for a MAC.

Appeared in State of the Art and Evolution of Computer Security and Industrial Cryptography, Lecture Notes in Computer Science 1528, Springer-Verlag, pp. 50-105. (C)1998 Springer-Verlag 
Just as for a MAC, the parties that use this approach are supposed to trust each other, but it is important to consider what will happen if a dispute arises, or what will happen if an insider attacks the system. An insider will try to find a collision, i.e., two plaintexts $X$ and $X^{\prime}$ such that $h(X)=h\left(X^{\prime}\right)$. Subsequently he will protect the authenticity of $X$ through $h(X)$, but at any time later he will be able to substitute $X^{\prime}$ for $X$. In order to avoid this attack, $h$ should be a CRHF.

However, one can certainly imagine applications where this attack is not relevant. In that case one only has to be protected against outsiders, hence it suffices that $h$ is a OWHF: an outsider cannot select $X$, but will only be able to observe $X$ and $h(X)$ and subsequently try to come up with an $X^{\prime}$ such that $h(X)=h\left(X^{\prime}\right)$.

1. The parties involved completely trust each other, which is trivially the case if there is only one party. One could think of someone who protects the integrity of his computer files through the calculation of an MDC that he stores in printed form in this vault. Every day he can repeat the calculation and verify the result.

2. The computation of the $h(X)$ involves a random component, that cannot be controlled by the insider (see Merkle [118] and Damgård [40]): $X$ can be randomized before applying $h$ through encryption of $X$ with a good block cipher using a truly random key, that is added to the resulting ciphertext [118], or through the selection of a short random prefix to $X$ [40]; $h$ itself can be randomized through randomly choosing $h$ from a family of functions indexed by a certain parameter (a UOWH as defined by Naor and Yung [128], cf. Sect. 3.2).

The advantage of a OWHF is that its design is easier and that storage for the hash result can be halved $(64 \ldots 80$ bits instead of $128 \ldots 160$ bits). However, note that the result cannot be too small as the security level degrades proportional to the number of applications of $h$ : an outsider who can attack a set $\{h(X) \mid X \in$ Domain $(h)\}$ of size $s$ has increased his probability to find an $X^{\prime}$ with a factor $s$. This limitation can be overcome through the use of a parameterized OWHF.

Note that one could also consider to encrypt an MDC in order to obtain a MAC (for example, [163, p. 622]). In this case the hash function has to be a CRHF, since a collision allows for a forgery after one chosen text. This approach imposes strong conditions on the encryption algorithm as well; for example, an additive stream cipher would obviously be insecure. For a block cipher in CBC mode, the security level is limited by the block size (the $I V$ can be used to manipulate the first ciphertext block). If the encryption algorithm (with the same key) is also used for encryption of plaintext, this solution is problematic, as a chosen text attack on the encryption scheme leads directly to a forgery of the MAC. In conclusion, this approach cannot be recommended as a general solution, as it abuses a primitive for secrecy to a primitive for authentication. A discussion of a related scheme (the secret suffix method) can be found in Sect. 7.2.

Appeared in State of the Art and Evolution of Computer Security and Industrial Cryptography, Lecture Notes in Computer Science 1528, Springer-Verlag, pp. 50-105. (C)1998 Springer-Verlag 
Authentication With Secrecy. If both authentication and secrecy are protected, the overall system can sometimes be simplified. For insider attacks, the additional encryption makes no difference, as an insider knows the secret key for the encryption. This means that for certain applications it should be hard to find collisions. For an outsider, an attack on the scheme becomes in general harder, as his knowledge decreases.

Although it is tempting to use this fact to lower the requirements imposed on the hash functions, this is certainly not a good practice. The additional protection offered by the encryption is dependent on the strength of the encryption algorithm and on the mode in which it is used.

MAC. Several options can be considered, but all share the problem of a double key management: one for the authentication and one for the encryption. They also require two passes over the message. It is tempting to use the same key twice, but this has to be discouraged strongly: not only are there dangerous interactions possible between the encryption scheme and the MAC algorithm, but the management of both keys should be different (e.g., lifetime, storage after use); finally, one risks that one application compromises the other one. The advantage of this approach is a high security level, owing to the complete separation of protection of privacy and authentication.

The most straightforward option is to calculate the MAC, append it to the information and subsequently encrypt the resulting message. This has the advantage that it precludes a direct exhaustive key search on the MAC key. An alternative is to omit the encryption of the MAC. The third solution is to calculate the MAC on the enciphered message; it has the advantage is that the authenticity can be verified without knowing the plaintext or the secret key of the encryption algorithm. This can be useful in the context of key management, but it is often preferable to protect the authenticity of the plaintext instead of the authenticity of the ciphertext. Recently Jakubowski and Venkatesan have provided a security proof for a construction that combines a simplified MAC algorithm with an additive stream cipher [92],

$M D C$. The advantages for using an MDC are a 'light weight' key management (only one key is required) and the fact that the authentication is derived directly from the privacy protection. Integrity and confidentiality can be protected at different layers in the communication protocol stack; no secret information is necessary at the (higher) layer that calculates the MDC. The disadvantage is that the protection of authenticity depends on the privacy protection: if the encryption algorithm is weak, the protection of authenticity will also be jeopardized. A second, more serious disadvantage is that such a solution is not acceptable with most standard encryption modes. If the MDC is calculated on the plaintext, and appended as the last block before the encryption, the following observations can be made:

- If the encryption algorithm is an additive stream cipher where the opponent knows the plaintext, an attack by Jueneman $[95,96]$ applies: an attacker can

Appeared in State of the Art and Evolution of Computer Security and Industrial Cryptography, Lecture Notes in Computer Science 1528, Springer-Verlag, pp. 50-105. (C)1998 Springer-Verlag 
easily compute the key-stream sequence. Subsequently he can modify the plaintext, calculate the MDC, and encrypt both the new plaintext and the new MDC. This attack is independent of the choice of the MDC. A solution suggested by Jueneman is to let the MDC depend on a random initial value $I V$, that depends on the previous message in the session. This approach is limited to session-based communications.

- Even if the encryption algorithm provides some error propagation, such as the CBC-mode of a block cipher, the scheme is vulnerable (this attack has been pointed out by D. Wagner): consider the plaintext $X\|\operatorname{MDC}(X)\| Y$, where $Y$ falls on a block boundary, and $\|$ denotes concatenation. An opponent who obtains the corresponding ciphertext can chop of the blocks corresponding to $Y$ and to the MDC on the plaintext, and obtain the ciphertext of an 'authentic' plaintext that was never sent (namely $X$ ). This particular attack can be precluded by prepending the length of the input. Randomization of the plaintext will not preclude this attack. A solution would be a very strong encryption mode, where every bit of the ciphertext depends in a complex way on every bit of the plaintext (for example the package transform of Rivest [152]). Simple variations of the CBC mode, such as the PCBC mode (which was used in Kerberos v4) are not sufficient.

Note that if the MDC is not encrypted, it will leak information. For example, anyone can verify whether the plaintext belongs to a small list of plaintexts, which is an undesirable property.

Because of the weaknesses pointed out, this solution is not recommended as a generic solution. Moreover, for large messages the unconditionally secure MACs (cf. Sect. 3.1) are more secure and faster than MDCs. The method should only be used in applications which cannot afford two independent strong operations. In that case some performance could be gained by using a function with weaker properties than an MDC; but is has been pointed that simple additions are not sufficient (see [134] for examples).

\section{An Overview of MDC Proposals}

First a general model is presented for iterated hash functions. Next four types of MDCs are briefly discussed: MDCs based on a block cipher, MDCs based on algebraic structures (modular arithmetic, knapsack, and lattice problems), and custom designed MDCs. For a more detailed discussion, the reader is referred to [134]. The performance in software of MDCs based on a block ciphers and custom designed hash functions is discussed by Preneel et al. in [138].

\subsection{A General Model for Iterated Hash Functions}

Before discussing a selection of the dozens of proposed MDCs, a general scheme for describing a hash function will be presented. Most known hash functions are based on a compression function with fixed size input; they process every message

Appeared in State of the Art and Evolution of Computer Security and Industrial Cryptography, Lecture Notes in Computer Science 1528, Springer-Verlag, pp. 50-105. (C)1998 Springer-Verlag 
block in a similar way. Lai and Massey call this an "iterated" hash function [107]. The information is divided into $t$ blocks $X_{1}$ through $X_{t}$. If the total number of bits is not a multiple of the block length, the information is padded to the required length (using a so-called padding rule). The hash function can then be described as follows:

$$
\begin{aligned}
H_{0} & =I V \\
H_{i} & =f\left(X_{i}, H_{i-1}\right) \quad i=1,2, \ldots t \\
h(X) & =g\left(H_{t}\right) .
\end{aligned}
$$

The result of the hash function is denoted with $h(X)$ and $I V$ is the abbreviation for Initial Value. The function $f$ is called the round function or compression function, and the function $g$ is called the output transformation. It is often omitted (that is, $g$ is often the identity function). Two elements in this definition have an important influence on the security of a hash function: the choice of the padding rule and the choice of the $I V$. It is recommended that the padding rule is unambiguous (i.e., there do not exist two messages that can be padded to the same padded message); at the end one should append the length of the message; and the $I V$ should be defined as part of the description of the hash function (this is called MD-strengthening after Merkle and Damgård). In some cases one can deviate from this rule, but this will make the hash function less secure and may lead to trivial collisions or second preimages.

Research on hash functions has been focussed on the question: which properties should be imposed on $f$ to guarantee that $h$ satisfies certain properties? Two partial answers have been found to this question. The first result is by Lai and Massey [107] and gives necessary and sufficient conditions for $f$ in order to obtain an "ideally secure" hash function $h$.

Theorem 1 (Lai-Massey). Assume that the padding contains the length of the input string, and that the message $X$ (without padding) contains at least 2 blocks. Then finding a second preimage for $h$ with a fixed $I V$ requires $2^{n}$ operations if and only if finding a second preimage for $f$ with arbitrarily chosen $H_{i-1}$ requires $2^{n}$ operations.

The fact that the condition is necessary is based on the following argument: if one can find a second preimage for $f$ in $2^{s}$ operations (with $s<n$ ), one can find a second preimage for $h$ in $2^{(n+s) / 2+1}$ operations with a meet-in-the-middle attack (cf. Sect. 6.2).

A second result by Damgård [42] and independently by Merkle [118] states that for $h$ to be a CRHF it is sufficient that $f$ is a collision resistant function.

Theorem 2 (Damgård-Merkle). Let $f$ be a collision resistant function mapping $l$ to $n$ bits (with $l-n>1$ ). If an unambiguous padding rule is used, the following construction yields a CRHF:

$$
\begin{aligned}
H_{1} & =f\left(0^{n+1} \| X_{1}\right) \\
H_{i} & =f\left(H_{i-1}\|1\| X_{i}\right) \quad \text { for } i=2,3, \ldots t .
\end{aligned}
$$

Appeared in State of the Art and Evolution of Computer Security and Industrial Cryptography, Lecture Notes in Computer Science 1528, Springer-Verlag, pp. 50-105. (C)1998 Springer-Verlag 
The construction can be improved slightly, and extended to the case where $l=$ $n+1$, at the cost of an additional assumption on $f$ (see [42] for details and Gibson's comment in [70]). It can also extended to a tree construction, which allows for increased parallelism [42].

\subsection{MDCs Based on a Block Cipher}

Several arguments can be indicated for designers of hash functions to base their schemes on existing encryption algorithms. The first argument is purely historical: DES [61] was the first standard commercial cryptographic primitive that was widely available; it seemed natural to construct hash functions based on this block cipher. A second argument is the minimization of the design and implementation effort: hash functions and block ciphers that are both efficient and secure are hard to design, and many examples that support this view can be found in the literature. Moreover, existing software and hardware implementations can be reused, which will decrease the cost. The major advantage however is that the trust in existing encryption algorithms can be transferred to a hash function. The main disadvantage of this approach is that custom designed hash functions are likely to be more efficient. This is particularly true because hash functions based on block ciphers require a key change after every encryption. Finally note that block ciphers may exhibit some weaknesses that are only important if they are used in a hashing mode (cf. Sect. 6.4). One also has to take into account that in some countries export restrictions for block ciphers may be tighter than those for hash functions.

The encryption operation $E$ will be written as $Y=E_{K}(X)$. Here $X$ denotes the plaintext, $Y$ the ciphertext, and $K$ the key. The corresponding decryption operation $D$ is written as $X=D_{K}(Y)$. The size of the plaintext and ciphertext or the block length (in bits) will be denoted with $r$, while the key size (in bits) will be denoted with $k$. In the case of the well known block cipher DES, $r=64$ and $k=56$ [61]. The hash rate of a hash function based on a block cipher is defined as the number of $r$-bit input blocks that can be processed with a single encryption.

A distinction will be made between the cases $n=r, n=2 \cdot r$, and $n>2 r$. This is motivated by the fact that most proposed block ciphers have a block length of only 64 bits, and hence an MDC with a result at least twice the block length is necessary to obtain a CRHF. Other proposals are based on a block cipher with a large key. Finally some related constructions are briefly discussed.

Size of Hash Result Equal to the Block Length. From Definition 2 it follows that these hash functions can only be collision resistant if the block length $r$ is at least 128 bits to 160 bits. Most present day block ciphers have only a block length of 64 bits, but the AES (Advanced Encryption Standard,) which NIST intends to publish by the year 2000, will have a block length of 128 bits.

Appeared in State of the Art and Evolution of Computer Security and Industrial Cryptography, Lecture Notes in Computer Science 1528, Springer-Verlag, pp. 50-105. (C)1998 Springer-Verlag 
All schemes of this type have rate 1 . The first secure construction for such a hash function was the ' 85 scheme by Matyas et al. [112]:

$$
H_{i}=E_{s\left(H_{i-1}\right)}^{\oplus}\left(X_{i}\right) .
$$

Here $s()$ is a mapping from the ciphertext space to the key space and $E_{K}^{\oplus}(X)$ denotes $E_{K}(X) \oplus X$. This is the main building block for all hash functions based on block ciphers. It is widely believed that this mapping is hard to invert, but there is no proof of this. One can apply the following intuitive reasoning: either one chooses the plaintext $X$, but then one has to find the key corresponding to one plaintext/ciphertext pair, which is deemed to be infeasible; alternatively, one chooses the key, but then one has to find for a given key a plaintext/ciphertext pair with a known difference, which is also believed to be difficult. This general construction is also used in several custom designed hash functions (cf. Sect. 5.4). This scheme has been included in ISO/IEC 10118-2 [90]. A variant on this scheme was proposed in 1989 independently by Preneel et al. [135] and by Miyaguchi et al. [124] for N-hash and later for any block cipher [91].

$$
H_{i}=E_{s\left(H_{i-1}\right)}^{\oplus}\left(X_{i}\right) \oplus H_{i-1} .
$$

Preneel et al. show that 12 secure variants exist, that are obtained by applying an affine transformation to the inputs of the two schemes above [137]. Moreover, the security level of these hash functions is limited by $\min (k, r)$, even if the size of some internal variables is equal to $\max (k, r)$. These variants have slightly different properties related to weak keys, the complementation property, and differential attacks (cf. Sect. 6). It is conjectured that for these schemes based on an 'ideal' block cipher (a random one-way permutation) no shortcut attacks exist, which implies that a collision attack requires about $2^{r / 2}$ operations and a preimage attack about $2^{r}$ operations.

An important variant of the first scheme is widely known as the Davies-Meyer scheme (the real inventors are probably Matyas and Meyer):

$$
H_{i}=E_{X_{i}}^{\oplus}\left(H_{i-1}\right) \text {. }
$$

It has the advantage that it extends more easily to block ciphers where key size and block size are different.

Size of Hash Result Equal to Twice the Block Length. The goal of double block length hash functions is to achieve a higher security level against collision attacks. Ideally a collision attack on such a hash function should require $2^{r}$ operations, and a (2nd) preimage attack $2^{2 r}$ operations.

A series of proposals attempted to double the size of the hash result, for example by iterating a OWHF; all of these succumbed to a 'divide and conquer' attack. A large class of proposals of rate 1 has the following form:

$$
\begin{aligned}
& H_{i}^{1}=E_{A_{i}^{1}}\left(B_{i}^{1}\right) \oplus C_{i}^{1} \\
& H_{i}^{2}=E_{A_{i}^{2}}\left(B_{i}^{2}\right) \oplus C_{i}^{2},
\end{aligned}
$$

Appeared in State of the Art and Evolution of Computer Security and Industrial Cryptography, Lecture Notes in Computer Science 1528, Springer-Verlag, pp. 50-105. (C)1998 Springer-Verlag 
where $A_{i}^{1}, B_{i}^{1}$, and $C_{i}^{1}$ are binary linear combinations of $H_{i-1}^{1}, H_{i-1}^{2}, X_{i}^{1}$, and $X_{i}^{2}$ and where $A_{i}^{2}, B_{i}^{2}$, and $C_{i}^{2}$ are binary linear combinations of $H_{i-1}^{1}, H_{i-1}^{2}, X_{i}^{1}$, $X_{i}^{2}$, and $H_{i}^{1}$. The hash result is equal to the concatenation of $H_{t}^{1}$ and $H_{t}^{2}$. First it was shown by Hohl et al. that the security level of the compression function of these hash functions is at most that of a single block length hash function [83]. Next Knudsen et al. showed that for all hash functions in this class, a preimage attack requires at most $2^{r}$ operations, and a collision attack requires at most $2^{3 r / 4}$ operations (for most schemes this can be reduced to $2^{r / 2}$ ) [101].

The few proposals that survive till today have rate less than 1 . Two important examples are MDC-2 and MDC- 4 with hash rate $1 / 2$ and $1 / 4$ respectively. They have been designed by Bracht et al. [24], and are also known as the MeyerSchilling hash functions after the authors of the first paper describing these schemes [122]. MDC-2 has been included in ISO/IEC 10118-2 [90] (in a more general form); it can be described as follows: The variables $H_{0}^{1}$ and $H_{0}^{2}$ are initialized with the

$$
\begin{array}{ll}
T_{i}^{1}=E_{u\left(H_{i-1}^{1}\right)}^{\oplus}\left(X_{i}\right)=L T_{i}^{1} \| R T_{i}^{1} & H_{i}^{1}=L T_{i}^{1} \| R T_{i}^{2} \\
T_{i}^{2}=E_{v\left(H_{i-1}^{2}\right)}^{\oplus}\left(X_{i}\right)=L T_{i}^{2} \| R T_{i}^{2} & H_{i}^{2}=L T_{i}^{2} \| R T_{i}^{1} .
\end{array}
$$

values $I V_{1}$ and $I V_{2}$ respectively, and the hash result is equal to the concatenation of $H_{t}^{1}$ and $H_{t}^{2}$. The ISO/IEC standard does not specify the block cipher; it also requires the specification of two mappings $u, v$ from the ciphertext space to the key space such that $u\left(I V^{1}\right) \neq v\left(I V^{2}\right)$. For DES, these mappings from 64 to 56 bits drop the parity bits in every byte and fix the second and third key bits to 01 and 10 respectively (to preclude attacks based on (semi-)weak keys). The best known preimage and collision attacks on MDC-2 require $2^{3 r}$ and $2^{r}$ operations respectively [107]. A collision attack on MDC-2 based on DES $(r=64, k=56)$ requires at most $2^{54}$ encryptions. However, it is obvious that the compression function of MDC-2 is rather weak: preimage and collision attacks on the compression function require at most $2^{r}$ and $2^{r / 2}$ operations (one fixes $X_{i}$ and varies $H_{i-1}^{1}$ and $H_{i-1}^{2}$ independently).

One iteration of MDC-4 consists of the concatenation of two MDC-2 steps, where the plaintexts in the second step are equal to $H 2_{i-1}$ and $H 1_{i-1}$. The rate of MDC-4 is equal to $1 / 4$. The best known attack to find a preimage for MDC-4 requires $2^{2 r}$ operations. This shows that MDC- 4 is probably more secure than MDC-2 against preimage attacks. However, a collision for the compression function of MDC-2 with a specified value for $H_{i-1}^{1}$ and $H_{i-1}^{2}$ also yields a collision for the compression function of MDC-4. Moreover, it has been demonstrated in by Preneel and Knudsen [134,102] that collisions for the compression function of MDC-4 can be found with $2^{3 r / 4}$ encryptions and the storage of $2^{3 r / 4} r$-bit quantities.

Merkle describes an interesting proposal in [118], for which he can prove that the compression function is collision resistant based on the assumption that the underlying single block length scheme is secure. The simplest scheme (with rate

Appeared in State of the Art and Evolution of Computer Security and Industrial Cryptography, Lecture Notes in Computer Science 1528, Springer-Verlag, pp. 50-105. (C)1998 Springer-Verlag 
1/18.3 for DES) can be described as follows:

$$
H_{i}=\operatorname{chop}_{16}\left[E_{0 \| H_{i-1}^{1}}^{\oplus}\left(H_{i-1}^{2} \| X_{i}\right) \| E_{1 \| H_{i-1}^{1}}^{\oplus}\left(H_{i-1}^{2} \| X_{i}\right)\right] .
$$

Here $H_{i-1}$ is a string consisting of 112 bits, the leftmost 55 bits of which are denoted $H_{i-1}^{1}$, and the remaining 57 are denoted $H_{i-1}^{2} ; X_{i}$ consists of 7 bits only. The function chop $r$ drops the $r$ rightmost bits of its argument. Note that this construction is similar to MDC-2 (but much slower). The most efficient proposal is more complex and use six invocations of the block cipher in two layers. Its hash rate is equal to 0.27 for DES. Merkle's proof for this proposal only showed a security level of $2^{52.5}$ against collisions; Preneel has improved this to $2^{56}$ [134].

Even the schemes in this class that provide optimal security do not offer long term collision resistance when used with DES; this will change with AES, which will have a block and key length of 128 bits (key lengths of 192 and 256 bits will also be provided).

Size of Hash Result Larger Than Twice the Block Length. Knudsen and Preneel also design a collision resistant compression function, but with parallel encryptions only [102]. They show how a class of efficient constructions for hash functions can be obtained by using non-binary error-correcting codes. Their schemes can achieve a provable security level against collisions equal to $2^{r}, 2^{3 r / 2}$ (or more) and this with rates larger than $1 / 2$; the security proof reduces the security of this scheme to an assumption on the single block length hash functions. The internal memory of the scheme is however much larger than 2 or 3 blocks, which implies that an output transformation is required. Earlier work by Preneel et al. [136] followed a related approach, but was not based on a collision resistant compression function.

Size of the Key Equal to Twice the Block Length. Some block ciphers have been proposed for which the key size is approximately twice the block length. One well known example is IDEA [106]. The AES variant with a 256-bit key also belongs to this class. It is not recommended to use triple DES with 2 keys here; one can exploit the structure in the triple DES key schedule.

Size of the Hash Result Equal to the Block Length. A scheme in this class was proposed by Merkle in [116] in '79, who observed that a block cipher with key size larger than block size is a natural compression function:

$$
H_{i}=E_{H_{i-1} \| X_{i}}(C),
$$

with $C$ a constant string. An alternative scheme was suggested by Lai and Massey [107]:

$$
H_{i}=E_{H_{i-1} \| X_{i}}\left(H_{i-1}\right) .
$$

These constructions can only yield a CRHF if the block length is larger than 128 bits (Merkle suggested 100 bits in 1979), and if the key size sufficiently large. For smaller block lengths, a OWHF can be obtained.

Appeared in State of the Art and Evolution of Computer Security and Industrial Cryptography, Lecture Notes in Computer Science 1528, Springer-Verlag, pp. 50-105. (C)1998 Springer-Verlag 
Size of the Hash Result Equal to Twice the Block Length. In order to obtain a CRHF based on a 64-bit block cipher, a different construction is required. The first two schemes in this class were proposed by Lai and Massey [107]. Both extend the Davies-Meyer scheme. One scheme is called "Tandem Davies-Meyer," and has the following description:

$$
\begin{aligned}
& H_{i}^{1}=E_{H_{i-1}^{2} \| X_{i}}^{\oplus}\left(H_{i-1}^{1}\right) \\
& H_{i}^{2}=E_{X_{i} \|\left(H_{i}^{1} \oplus H_{i-1}^{1}\right)}^{\oplus}\left(H_{i-1}^{2}\right) .
\end{aligned}
$$

The second scheme is called "Abreast Davies-Meyer":

$$
\begin{aligned}
& H_{i}^{1}=E_{H_{i-1}^{2} \| X_{i}}^{\oplus}\left(H_{i-1}^{1}\right) \\
& H_{i}^{2}=E_{X_{i} \| H_{i-1}^{1}}^{\oplus}\left(\bar{H}_{i-1}^{2}\right),
\end{aligned}
$$

Here $\bar{H}$ denotes the bitwise complement of $H$. Both schemes have rate equal to $1 / 2$; the best known attacks for a preimage and a collision requires $2^{2 r}$ respectively $2^{r}$ encryptions. Faster schemes in this class have been developed in [102].

Related schemes. Aiello and Venkatesan propose in [4] a construction to double the output of a random function. In order for it to be usable for hashing, one needs to define the key schedule of this larger 'block cipher'. The construction by Aiello, Haber, and Venkatesan [5] replaces the key schedule of DES by a function from the MDx-family (cf. Sect. 5.4); several instances are combined by choosing different (fixed) plaintexts.

\subsection{MDCs Based on Algebraic Structures}

First hash functions based on modular arithmetic are considered. Next hash functions based on knapsack problems and lattices are presented. This section is concluded with a short discussion of incremental hash functions.

MDCs Based on Modular Arithmetic. These hash functions are designed to use the modular arithmetic hardware that is required to produce digital signatures (for example, RSA [153]). The security of certain constructions can be based on the hardness of some number theoretic problems. Moreover these schemes are easily scalable. The disadvantage is that the underlying primitive has a rich mathematical structure; this can be exploited in attacks that use the homomorphic structure and the fixed points of modular exponentiation (trivial examples are 0 and 1 ); one also has to ensure that no small inputs occur.

A distinction is made between 'ad hoc' schemes, which have no provable reduction to the underlying hard problem, and provably secure schemes. Schemes in the first class are typically much more efficient, but many proposals have been broken; however, it seems that recently designers have been more conservative and designs survive longer.

Appeared in State of the Art and Evolution of Computer Security and Industrial Cryptography, Lecture Notes in Computer Science 1528, Springer-Verlag, pp. 50-105. (C)1998 Springer-Verlag 
Schemes Without Security Reduction. Most of these schemes uses a modulus $N$, that is, the product of two large primes. The size of $N$ in bits (denoted with $n$ ) is typically between 512 and 1024. These hash functions can be useful in combination with RSA [153] as digital signature scheme. However, this choice poses the following practical problem: the person who has generated the modulus knows its factorization, and hence he has a potential advantage over the other users of the hash function. One can try to design the hash function such that knowledge of the factorization does not help in attacking it (this is probably difficult to achieve). Alternatives are to use a trusted third party to generate the modulus (for example, the modulus of the Certification Authority), or to generate the modulus using a multi-party secure computation; recently practical solutions for such a computation have been developed by Boneh and Franklin [22] and Frankel et al. [67].

The most efficient schemes are based on modular squaring. An additional argument for squaring is that any algorithm that can extract modular square roots is reducible to a factoring algorithm (in random polynomial time). One approach is to study all possible schemes that use a single squaring and exclusive ors and that have an internal memory of only one block. The best scheme in this class seems to be of the form: $H_{i}=\left(X_{i} \oplus H_{i-1}\right)^{2} \bmod N \oplus H_{i-1}$. The introduction of the feedforward offers certainly advantages over the CBC-mode, which can be found in many earlier proposals.

It is essential to add redundancy to the message input. However, Girault [73] and Girault and Misarsky [75] have pointed out that early proposals to insert a fixed pattern (for example, zeroes in the beginning, middle, or end) are insecure. This has resulted in dispersing the redundancy to the most significant nibble (4 bits) of every byte. This proposal made it to the informative annex D of CCITT-X.509:1989 [29]. Coppersmith showed however that one can construct two messages such that their hash results are a multiple of each other [35]. If the hash function is combined with a multiplicative signature scheme like RSA [153], one can exploit this attack to forge signatures. As a consequence, new methods for adding redundancy were proposed within ISO/IEC SC27. The current version of the Final Draft International Standard (FDIS) is called MASH-1 (for Modular Arithmetic Secure Hash) [90]:

$$
H_{i}=\left(\left(X_{i} \oplus H_{i-1}\right) \vee A\right)^{2} \quad(\bmod N) \oplus H_{i-1}
$$

here $A=0 \mathrm{xF00} \ldots 00$, the four most significant bits in every byte of $X_{i}$ are set to 1111 , and the output of the squaring operation is chopped to $n$ bits. A complex output transformation is added, which consists of a number of applications of the compression function; its goal is to destroy all the remaining mathematical structure. The final result is at most $n / 2$ bits. The best known preimage and collision attacks on MASH-1 require $2^{n / 2}$ and $2^{n / 4}$ operations; they are thus not better than brute force attacks.

Stronger schemes have been proposed that are slower. Examples are the use of two squaring operations [73]: $f=\left(H_{i-1} \oplus\left(X_{i}\right)^{2}\right)^{2} \bmod N$, and the replacement of the squaring by a higher exponent (typically $\left.3 \ldots 2^{16}+1\right)$. For example,

Appeared in State of the Art and Evolution of Computer Security and Industrial Cryptography, Lecture Notes in Computer Science 1528, Springer-Verlag, pp. 50-105. (C)1998 Springer-Verlag 
MASH-2 is a variant of MASH- 1 which uses exponent $2^{8}+1$ [90]. This provides for an additional security margin.

Schemes With a Security Reduction. For several schemes there exists a security reduction to a number theoretic problem that is believe to be difficult. However, they are very slow: typically they hash $\log _{2} \log _{2} N$ bits per modular squaring (or even per modular exponentiation).

Damgård provides two hash functions for which finding a collision is provably equivalent to factoring an RSA modulus [40]. Gibson proposes a construction based on the discrete logarithm problem modulo a composite [71]. A third approach uses the discrete logarithm problem in a group of prime order $p$ denoted with $G_{p}$ (Bellare et al. [12], after earlier work by Chaum et al. [33] and Brands). Every non-trivial element of $G_{p}$ is a generator. The hash function uses $t$ random elements $\alpha_{i}$ from $G_{p}\left(\alpha_{i} \neq 1\right)$. The hash result is then computed as

$$
H_{t+1}=\prod_{i=1}^{t} \alpha_{i}^{\tilde{X}_{i}}
$$

Here $\tilde{X}_{i}$ is obtained by considering the string $X_{i}$ as the binary expansion of a number and prepending 1 to it. This avoids trivial collisions when $X_{i}$ consists of all zeroes.

MDCs Based on Knapsack and Lattice Problems. The knapsack problem (which is a special case of the subset sum problem) of dimensions $n$ and $\ell(n)$ can be defined as follows: given a set of $n l$-bit integers $\left\{a_{1}, a_{2}, \ldots, a_{n}\right\}$, and an integer $S$, find a vector $X$ with components $x_{i}$ equal to 0 or 1 such that

$$
\sum_{i=1}^{n} a_{i} \cdot x_{i}=S \bmod 2^{\ell(n)} .
$$

For application to hashing, one needs $n>\ell(n)$. The knapsack problem is known to be NP-hard; while this means that probably no feasible worst-case algorithms for this problem exists, this does not tell much about the hardness of a random instance. This problem was used in 1978 by Merkle and Hellman to construct the first public-key encryption system [120]. However, almost all public key schemes based on the knapsack problem have been broken (see for example [129]), which has given the knapsack a bad reputation. The appeal of the knapsack problem (and related lattice based problems) lies in the fact that both hardware and software implementations are very fast compared to schemes based on number theoretic problems. Moreover, evaluation of a knapsack allows for significant parallelism. Finally, interesting security reductions can be proved: examples are the work for Impagliazzo and Naor [84] on knapsacks and that of Ajtai [6] for lattices; Ajtai was able to prove that if the shortest vector in a lattice problem is hard in the worst case, then the knapsack problem is hard on the average. However, some researchers believe that for realistic parameters, both these problems

Appeared in State of the Art and Evolution of Computer Security and Industrial Cryptography, Lecture Notes in Computer Science 1528, Springer-Verlag, pp. 50-105. (C)1998 Springer-Verlag 
are relatively easy. If they are right, knapsack and lattice problems are not useful to practical cryptography.

Attacks on knapsacks often use the LLL lattice reduction algorithm [108] that finds the shortest vector in a lattice (the algorithm performs in practice much better than can be guaranteed). This reduction to the shortest vector problem only works for $\ell(n)>1.0629 \cdot n$. Knapsack problems become more difficult when $n \approx \ell(n)$; however, the performance of the hash function decreases with the value $n-\ell(n)$. For $n=\ell(n)$, the best known attack requires time $O\left(2^{n / 2}\right)$ and space $O\left(2^{n / 4}\right)$. Impagliazzo and Naor summarize the state of the art in [84]. A different class of attacks are the algebraic attacks proposed by Camion and Patarin [26] and optimized by Patarin in [132]; these attacks tend to work better when $n \gg \ell(n)$. The scheme of Damgård [42] has been broken both using LLL [94] and using algebraic techniques [132]. It is for the time being an open problem whether a random knapsack with $n=1024, l=512$, and $\ell=512$ is hard to solve.

Impagliazzo and Naor describe an efficient construction for a UOWHF (cf. Sect. 3.2) and provide a reduction of its security to that of the knapsack problem [84]. Ajtai introduced a function that is one-way (or preimage resistant) if the problem of approximating the shortest vector in a lattice to polynomial factors is hard [6]. Goldreich et al. have proved that the function is in fact collision resistant.

Several multiplicative knapsacks have also been proposed; multiplicative notation is used for non-abelian groups. The earliest proposal dates back to ' 77 (but it was quickly shown to be insecure). A recent example are the schemes by Zémor [174] and Tillich and Zémor [168]. Their security is based on the hardness of finding short factorizations in certain groups. In some cases one can even prove a lower bound on the Hamming distance between colliding messages. Attacks on these proposals (for certain parameters) can be found in [31,69]. Impagliazzo and Naor also extend their construction on a UOWHF to multiplication in a group [84].

Knapsack and lattice based hash functions have also the potential problem that trapdoors may be inserted when the vectors are generated. Therefore it is recommended that the instance generation is reproducible (for example, through the use of a pseudo-random string generator or a hash function).

Incremental Hash Functions. A hash function (or any cryptographic primitive) is called incremental if it has the following property: if the hash function has been evaluated for an input $x$, and a small modification is made to $x$, resulting in $x^{\prime}$, then one can update $h(x)$ in time proportional to the amount of modification between $x$ and $x^{\prime}$, rather than having to recompute $h\left(x^{\prime}\right)$ from scratch. If a function is incremental, it is automatically parallelizable as well.

This concept was first introduced by Bellare et al. [12]. They also made a first proposal based on exponentiation in a group of prime order. Improved constructions were proposed by Bellare and Micciancio [15] that consist of two steps:

Appeared in State of the Art and Evolution of Computer Security and Industrial Cryptography, Lecture Notes in Computer Science 1528, Springer-Verlag, pp. 50-105. (C)1998 Springer-Verlag 
- First the message is divided into blocks; each block (together with its index) is hashed using a conventional hash function (restricted to fixed length inputs). This is called the 'randomization' step as in the analysis the hash function is treated as an 'ideal' hash function or random oracle (which is a very demanding requirement).

- Next the different outputs are combined using a group operation. This can be a group of large prime order in which the discrete logarithm problem is hard, and modular addition. The first approach leads to a reduction to the discrete logarithm problem, while the second leads to a reduction to the 'weighted knapsack' problem.

The same techniques can also be used to improve the lattice based hash function. These schemes have the advantage that they are much more efficient than the other schemes studied in this section. However, this comes at a cost of requiring a collision resistant hash function, which also has to behave 'perfectly random.' This construction is remarkable, as it construct a collision resistant function based on a one-way property (but with specific algebraic structure, so there is no contradiction to the result of Simon [164] discussed at the end of Sect. 3.2).

\subsection{Custom Designed MDCs}

This section discusses a selection of custom designed hash functions, i.e., algorithms that were especially designed for hashing operations. Most of these algorithms use the Davies-Meyer approach (cf. Sect. 5.2): the compression function is a block cipher, 'keyed' by the text input $X_{i}$; the plaintext is the value $H_{i-1}$, which is also added to the ciphertext (feedforward).

MD2 [98] is a hash function with a 128-bit result that was published by Rivest of RSA Data Security Inc. in 1990. The algorithm is software oriented, but due to the byte structure it is not very fast on 32-bit machines. It inserts at the end a checksum byte (a weak hash function) of all the inputs. Rogier and Chauvaud find collisions for a variant of MD2, that omits the checksum byte at the end [155].

A much faster algorithm by the same designer is MD4 [150]; it also dates back to 1990 and has a 128-bit result. The main merit of the algorithm is that it introduces innovative design principles; the most important one is the use of 32-bit integer arithmetic. The algorithms derived from it (with improved strength) are often called the MDx-family. This family contains the most popular hash functions in use today. Dobbertin has found collisions for MD4; his attack combines algebraic techniques and optimization techniques such as genetic algorithms [51, 52]. It can be extended in such a way that even 'meaningful' collision are obtained: the complete message (except for a few dozen bytes) is under complete control of the attacker. His attack also applies to the compression function of 'extended MD4' [150], which consists of concatenating two loosely coupled instances of MD4. Later Dobbertin showed that a reduced version of MD4 (2 rounds out of 3) is not preimage resistant [54].

Appeared in State of the Art and Evolution of Computer Security and Industrial Cryptography, Lecture Notes in Computer Science 1528, Springer-Verlag, pp. 50-105. (C)1998 Springer-Verlag 
Following early attacks on MD4 by Merkle and den Boer and Bosselaers [47], Rivest quickly proposed a strengthened version, namely MD5 [151]. It was however shown by den Boer and Bosselaers [48] that the compression function of MD5 is not collision resistant (but their collisions are of the form $f\left(H_{i-1}, X_{i}\right)=$ $f\left(H_{i-1}^{\prime}, X_{i}\right)$, which is not immediately usable in practice). Dobbertin has extended his attack on MD4 to yield collisions for the compression function of MD5, i.e., $f\left(H_{i-1}, X_{i}\right)=f\left(H_{i-1}, X_{i}^{\prime}\right)$, where he has some control over $H_{i-1}$ [53]. It is believed that it is feasible to extend this attack to collisions for MD5 itself (that is, to take into account the $I V$ ). HAVAL was proposed by Zheng, Pieprzyk, and Seberry at Auscrypt'92 [176]; it consists of several variants, and is an extension of MD5.

A second improved variant of MD4, the Secure Hash Algorithm, was proposed by NIST [64] in 1993. The size of the hash result is increased from 128 to 160 bits and the message words are not simply permuted but encoded with a shortened cyclic code. After a few years, NSA discovered a certificational weakness in SHA; apparently collisions can be found in less than $2^{80}$ operations. Consequently a new release of the standard was published. The new algorithm is called SHA-1 [65]. Recently Chabaud and Joux have published an attack that finds collisions for SHA in $2^{61}$ operations [30]; it is probably similar to the (classified) attack developed earlier that prompted the upgrade to SHA-1.

Yet another improved version of MD4, called RIPEMD, was developed in the framework of the EEC-RACE project RIPE [149]. Due to partial attacks by Dobbertin [51], it was later upgraded by Dobbertin et al. to RIPEMD-128 and RIPEMD-160, that have a 128-bit and a 160-bit result respectively [55]. Variants with a 256 and 320-bit result have been introduced as well. Together with SHA1, RIPEMD-128 and RIPEMD-160 are the three custom designed hash functions included in ISO/IEC 10118-3 [90].

N-hash is a hash function designed by Miyaguchi et al. [124,125]. den Boer (unpublished) and Biham and Shamir [19] have identified serious weaknesses in this scheme. A new version of N-hash appeared in a Japanese contribution to ISO [91]. It was shown that this scheme contains additional weaknesses [18, 134].

Schnorr and Vaudenay propose in [159] FFT-Hash III; as the name suggests, Schnorr has made some earlier designs that were shown to be weak. No weaknesses have been reported for FFT-Hash III.

Merkle suggested in 1989 a software oriented one-way hash function called Snefru [119]. It is based on large random substitution tables (2 Kbyte per pass) and allows for a variable size of the result (128 or 256 bits). Biham and Shamir have shown that Snefru-128 [19] is vulnerable to differential attacks. As a consequence it is recommended to use 6 and preferably 8 passes, preferably with a 256-bit hash result. However, these measures increase the size of the substitution tables and decrease the performance [138].

Two of the most recent designs are Tiger and Panama. Tiger was proposed by Anderson and Biham [7]. It is tuned towards 64-bit processors and mixes Snefru-type S-boxes (8 input bits and 64 output bits) with arithmetic operations.

Appeared in State of the Art and Evolution of Computer Security and Industrial Cryptography, Lecture Notes in Computer Science 1528, Springer-Verlag, pp. 50-105. (C)1998 Springer-Verlag 
Panama is a design of Daemen and Clapp [38]; it is designed to take advantage of the instruction-level parallelism in modern processors.

The scheme by Damgård [42] based on a cellular automaton was broken by Daemen et al. in [39]. In the same paper these authors have proposed Cellhash, a new hash function based on a cellular automaton [39]. Later an improved version called Subhash was published [37]. Both schemes are hardware oriented.

\section{Methods of Attack on MDCs}

This section gives an overview of the known methods of attack on MDCs. This taxonomy can be helpful to understand the attacks published in the literature, but can also serve as a caveat for designers or evaluators. From these attacks, one can conclude that one should take a conservative approach. This implies that if one can find 'randomly looking' collisions or second preimages for an MDC, one should anticipate that a further refinement or optimization of the attack can find collisions or second preimages that satisfy additional constraints.

\subsection{Attacks Independent of the Algorithm}

These attacks depend only on the size $n$ in bits of the hash result, and are independent of the specific details of the algorithm. It is assumed that the MDC approximates a random function: if this is not the case this class of attacks will be even more successful. For the time being $2^{64}$ operations is considered to be on the edge of feasibility. In view of the fact that the speed of computers is multiplied by four every three years (this is one of the formulations of Moore's law), $2^{70}$ operations is sufficient for the next 5 to 10 years, but it will be only marginally secure within 15 years. For applications that require protection for 20 years, one should try to design the scheme such that an attack requires at least $2^{80}$ operations.

Random (2nd) Preimage Attack. The opponent selects a random message and hopes that a given hash result will be hit. If the hash function has the required "random" behavior, his probability of success equals $1 / 2^{n}$ with $n$ the number of bits of the hash result. This attack can be carried out off-line and in parallel, which means that $n$ should be at least 64 . If a significant number of messages can be attacked simultaneously, it is advisable to select a larger value of $n$.

Birthday Attack. The birthday paradox states that for a group of 23 people, the probability that at least two people have a common birthday exceeds $1 / 2$. Intuitively one expects that the probability is much lower. However, the number of pairs of people in such a group equals $23 \cdot 22 / 2=253$. This can be exploited to attack a hash function in the following way: an adversary generates $r_{1}$ variations

Appeared in State of the Art and Evolution of Computer Security and Industrial Cryptography, Lecture Notes in Computer Science 1528, Springer-Verlag, pp. 50-105. (C)1998 Springer-Verlag 
on a bogus message and $r_{2}$ variations on a genuine message. The expected number of collisions equals $r_{1} \cdot r_{2} / n$. The probability of finding a bogus message and a genuine message that hash to the same result is given by $1-\exp \left(-r_{1} \cdot r_{2} / 2^{n}\right)$, which is about $63 \%$ when $r=r_{1}=r_{2}=2^{\frac{n}{2}}$. Finding the collision does not require $r^{2}$ operations: after sorting the data, which requires $O(r \log r)$ operations, comparison is easy. This attack was first pointed out by Yuval [173].

One can reduce the memory requirements for collision search by translating the problem to the detection of a cycle in an iterated mapping. Indeed, any mapping that is iterated on a finite set will eventually repeat, i.e., it will enter a cycle. If the mapping is a random mapping (rather than a permutation), the entry point to the cycle corresponds to a collision for the function (unless the starting point belonged to the cycle, but this event has a negligible probability). The detection of a cycle does not require storing all the values; for example, the technique of distinguished points can be used (one only stores special points, for example those points beginning with 30 zero bits). Cycle detection techniques were first applied to collision search by Quisquater [143]. The expected number of function evaluations of his algorithm is about $2 \sqrt{\pi / 2} \cdot 2^{\frac{n}{2}}$; the storage requirements are negligible. In [169], van Oorschot and Wiener propose an efficient parallel variant of this algorithm: the speed-up is linear with the number of processors. They estimate that with a 10 million US\$ machine, collisions for MD5 (with $n=128$ ) can be found in 21 days (in 1994). In order to make a collision search infeasible, $n$ should be at least 160 bits. This explains the second condition in Definition 2 of a CRHF.

For digital signatures, hash functions need to be collision resistant since otherwise one can sign one message and later claim to have signed a different message, or be held accountable for a different message. There is no way to prevent a sender from performing this attack, although the occurrence of two messages that hash to the same value might make him suspect (in practice it might be very difficult to prove to a third party the denial). Outsiders can perform the same attack if they can convince the signer to sign a message of their choice. The sender can protect himself through randomizing the message just prior to signing (or by randomizing the hash function as is done for a UOWHF, cf. Sect. 3.2).

\subsection{Attacks Dependent on the Chaining}

This class of attacks depends on some high level properties of the compression function $f$.

Meet-in-the-Middle Attack. This attack is a variation on the birthday attack, but instead of comparing the hash result, one compares intermediate chaining variables. The attack enables an opponent to construct a (2nd) preimage, which is not possible for a simple birthday attack. The opponent generates $r_{1}$ variations on the first part of a bogus message and $r_{2}$ variations on the last part. Starting from the initial value and going backwards from the hash result, the probability for a matching intermediate variable is again given by

Appeared in State of the Art and Evolution of Computer Security and Industrial Cryptography, Lecture Notes in Computer Science 1528, Springer-Verlag, pp. 50-105. (C)1998 Springer-Verlag 
$1-\exp \left(-r_{1} \cdot r_{2} / 2^{n}\right)$. The only restriction that applies to the meeting point is that it cannot be the first or last value of the chaining variable. The cycle finding algorithm has been extended by Quisquater to perform a meet-in-themiddle attack with negligible storage [144]. The attack can be precluded by avoiding functions $f$ that are invertible to the chaining variable $H_{i-1}$ and to the message $X_{i}$ (see also Theorem 1 in Sect. 5.1).

Further extensions of this attack have been proposed by Coppersmith [34] and Girault et al. [74] to break $p$-fold iterated schemes, i.e., weak schemes with more than one 'pass' over the message as proposed by Davies [44]. Other extensions take into account additional constraints on the message.

Correcting Block Attack. This attack consists of substituting all blocks of the message except for one or more blocks. This attack often applies to the last block and is then called a correcting last block attack, but it can also apply to the first block or to some blocks in the middle. For a (2nd) preimage attack, one chooses an arbitrary message $X$ and finds one or more correcting blocks $Y$ such that $h(X \| Y)$ takes a certain value. For a collision attack, one starts with two arbitrary messages $X$ and $X^{\prime}$ and appends one or more correcting blocks denoted with $Y$ and $Y^{\prime}$, such that the extended messages $X \| Y$ and $X^{\prime} \| Y^{\prime}$ have the same hash result. The hash functions based on modular arithmetic are especially sensitive to this attack.

One can try to preclude a correcting block attack by adding redundancy to the message blocks, in such a way that it becomes computationally infeasible to find a correcting block with the necessary redundancy. The price paid for this solution is a degradation of the performance.

Fixed Point Attack. The idea of this attack is to look for a $H_{i-1}$ and $X_{i}$ such that $f\left(X_{i}, H_{i-1}\right)=H_{i-1}$. If the chaining variable is equal to $H_{i-1}$, it is possible to insert an arbitrary number of blocks equal to $X_{i}$ without modifying the hash result. Producing collisions or a second preimage with this attack is only possible if the chaining variable can be made equal to $H_{i-1}$ : this is the case if $I V$ can be chosen equal to a specific value, or if a large number of fixed points can be constructed (e.g., if one can find an $X_{i}$ for a significant fraction of $H_{i-1}$ 's). Of course this attack can be extended to fixed points that occur after more than one iteration. This attack can be made more difficult by appending a block count and by fixing $I V$ (MD-strengthening, see Sect. 5.1).

Differential Attacks. Differential cryptanalysis studies the relation between input and output differences and is applicable to both block ciphers and hash functions $[18,19]$. Interesting results were obtained by Biham and Shamir for FEAL, DES, N-hash, and Snefru. Differential attacks of hash functions based on block ciphers have been studied by Preneel and Rijmen [134,148].

Analytical Weaknesses. Some MDCs allow manipulations as insertion, deletion, permutation, and substitutions of blocks. A large number of attacks have

Appeared in State of the Art and Evolution of Computer Security and Industrial Cryptography, Lecture Notes in Computer Science 1528, Springer-Verlag, pp. 50-105. (C)1998 Springer-Verlag 
been based on blocking the diffusion of the data input: this means that changes have no effect or can be canceled out easily in a next stage. This type of attacks has been successful for custom designed hash functions and for hash functions based on modular arithmetic. Among the most remarkable attacks in this class are the attacks developed by Dobbertin on the MDx-family [51,52]. They combine conventional optimization techniques (simulated annealing, genetic algorithms) with conventional cryptanalytic techniques. Another property of the attacks is that differences are only controlled in certain points of the algorithms; this in contrast to differential cryptanalysis, where typically all intermediate differences are controlled to a certain extent.

\subsection{Attacks Dependent on Interaction with the Signature Scheme}

Even if the hash function is collision resistant, it might be possible to break the signature scheme. This attack is then the consequence of an interaction between both schemes. In the known examples of such an interaction both the hash function and the signature scheme have some multiplicative structure (Coppersmith's attack on X.509 Annex D [35]). Damgård has proved that the security of a digital signature scheme which is not existentially forgeable under a chosen message attack will not decrease if it is combined with a CRHF [40] (cf. Sect. 9.4).

A more subtle point is that problems can arise if the hash function and the digital signature scheme are not coupled. For example, given $h(X)$, with $h$ a strong CRHF, one could try to find a value $X^{\prime}$ such that $h^{\prime}\left(X^{\prime}\right)=h(X)$, where $h^{\prime}$ is a 'weak' hash function, and then claim that the signer has signed $X^{\prime}$ with $h^{\prime}$ instead of $X$ with $h$. This problem can be solved by signing together with the hash result a unique hash identifier (for example, as defined in [90]), or by allowing only one hash function for a given signature scheme (DSA [66] and SHA-1 [65]).

\subsection{Attacks Dependent on the Underlying Block Cipher}

Certain weaknesses of a block cipher are not significant if the block cipher is used for encryption, but can have dramatic consequences if it is used in one of the modes for hashing. These weaknesses can be exploited to insert special messages or to carry out well chosen manipulations without changing the hash result. We will only discuss the weaknesses of DES [61], because of its widespread use.

Complementation Property. One of the first properties that was known of DES was the symmetry under complementation [82]: $\forall P, K: C=\operatorname{DES}(K, P)$ $\Longleftrightarrow \bar{C}=\operatorname{DES}(\bar{K}, \bar{P})$. It can reduce an exhaustive key search by a factor 2 but it also allows to construct trivial collisions.

Weak Keys. Another well known property of DES is the existence of four weak keys $[46,126]$. For these keys, encryption equals decryption, or DES is an involution. These keys are also called palindromic keys. This means that

Appeared in State of the Art and Evolution of Computer Security and Industrial Cryptography, Lecture Notes in Computer Science 1528, Springer-Verlag, pp. 50-105. (C)1998 Springer-Verlag 
$E_{K}\left(E_{K}(P)\right)=P, \forall P$. There exist also 6 pairs of semi-weak keys, for which $E_{K_{2}}\left(E_{K_{1}}(P)\right)=P, \forall P$. This property can be exploited in certain hash functions to construct fixed points after two iterations steps.

Knudsen has identified other classes of weak keys [99], namely weak hash keys and quasi weak keys. Quasi weak keys are keys for which there exists a simple relation between the corresponding encryption functions; DES has such keys which are not weak keys.

Fixed Points. Fixed points of a block cipher are plaintexts that are mapped to themselves for a certain key. As a secure block cipher is a random permutation, it will probably have fixed points (for every key there is a probability of $1-e^{-1}$ that there is at least a single fixed point). However, it should be hard to find these. For DES, it was pointed out that finding fixed points is easy for some keys [126]: for every weak key $K_{p}$, there exist $2^{32}$ values of $P$ that can be easily found for which $\operatorname{DES}\left(K_{p}, P\right)=P$. A similar property holds for the anti-palindromic keys: these are 4 semi-weak keys for which there exist $2^{32}$ values of $P$ that can be easily found for which $\operatorname{DES}\left(K_{a p}, P\right)=\bar{P}$.

Key Collisions. The collision search algorithm discussed in Sect. 6.1 can also be used to construct key collisions for a block cipher. A key collision is a pair of keys $K_{1}, K_{2}$ such that $E_{K_{1}}(P)=E_{K_{2}}(P)$ for a plaintext $P$. For DES, there exist about $\left(2^{56}\right)^{2} / 2^{65}=2^{47}$ collisions for a given $P$. Finding one collision requires about $2^{r / 2}$ encryptions and very little storage, which is feasible for any block cipher with $r<128 \ldots 160$. However, a good design of the hash function can make the collisions useless. There is no easy way to guarantee this, and every scheme has to be verified for this attack.

\section{An Overview of MAC Algorithm Proposals}

In contrast with the variety of MDC proposals, very few MAC algorithms exist. The main reason is perhaps the fact that the existing standards are widely accepted.

The general model for an iterated MAC algorithm is similar as the model for an MDC (cf. Sect. 5.1). The main difference is that the compression function $f$ and in some cases the initial value $I V$ depend on the secret key $K$. Moreover, an output transformation is frequently used. Bellare et al. prove that iterating a finite size pseudo-random function provides a pseudo-random function that can take an input of arbitrary size [11].

Three types of MAC algorithms are discussed: MAC algorithms based on block ciphers, MAC algorithms based on hash functions, and dedicated MAC algorithms. The performance in software of the first two type of MAC algorithms is discussed by Preneel et al. [138]. Note that if one is willing to use longer keys or update the key per message, one should consider the unconditionally secure schemes discussed in Sect. 3.1; the fastest of these schemes are significantly faster than the fastest MDCs and MACs.

Appeared in State of the Art and Evolution of Computer Security and Industrial Cryptography, Lecture Notes in Computer Science 1528, Springer-Verlag, pp. 50-105. (C)1998 Springer-Verlag 


\subsection{MAC Algorithms Based on a Block Cipher}

The most popular method to compute a MAC is CBC-MAC, which uses the Cipher Block Chaining mode of a block cipher with $I V=H_{0}=0$. The scheme appeared already in a 1977 document by Campbell [27]; it has been included in several standards: ANSI X9.9 [8], ANSI X9.19 [9], FIPS 113 [63], ISO 8731 [86], and ISO/IEC 9797 [88].

$$
H_{i}=E_{K}\left(H_{i-1} \oplus X_{i}\right) .
$$

Note that one can obtain the same output by using the Cipher FeedBack (CFB) mode, provided that an extra iteration is performed at the end. The standards differ because some of them mandate the use of DES as the block cipher, select one of the two modes, suggest other padding schemes, or leave open the number $m$ of output bits.

CBC-MAC is vulnerable to the so-called exor forgery attack that requires only one known text-MAC pair (cf. Sect. 8.2). This attack can be precluded by a strong output transformation (just chopping off some bits may not be enough).

A good example of an output transformation is the encryption of the value $H_{t}$ with a key derived from $K$ (cf. ISO/IEC 9797 [88] and [149]). For DES, an even better alternative is to replace the processing of the last block by a two-key triple encryption (with keys $K_{1}=K$ and $K_{2}$ ); this is commonly known as the ANSI retail MAC, since it first appeared in ANSI X9.19 [9]:

$$
g\left(H_{t}\right)=E_{K_{1}}\left(D_{K_{2}}\left(H_{t}\right)\right) .
$$

This mapping requires little overhead, and has the additional advantage that it precludes an exhaustive search against a 56-bit DES key; it also protects against theoretical shortcut attacks on DES (such as differential [19] and linear [109] attacks).

Preneel and van Oorschot have shown that for the ANSI retail MAC, $2^{r / 2}$ known texts allow for a key recovery in only $3 \cdot 2^{k}$ encryptions, compared to $2^{2 k}$ encryptions for exhaustive key search [142] (note that for DES $r=64$ and $k=56$ ). Another key recovery attack needs only a single known text, but requires about $2^{k}$ MAC verifications [103]. Moreover, it reduces the effective MAC size from $\min (r, 2 k)$ to $\min (r, k)$. Apparently, the security of the ANSI retail MAC can be improved at no cost in performance by introducing a double DES encryption in the first and last iteration [103]:

$$
H_{1}=E_{K_{2}^{\prime}}\left(E_{K_{1}}\left(X_{1}\right)\right) \text { and } g\left(H_{t}\right)=E_{K_{2}}\left(H_{t}\right) .
$$

Here $K_{2}^{\prime}$ is derived from $K_{2}$.

A new mode to compute a MAC was suggested by Preneel $[134,149]$ (called RIPEMAC):

$$
H_{i}=E_{K}\left(X_{i} \oplus H_{i-1}\right) \oplus X_{i} .
$$

It has the advantage that the compression function is harder to invert, even for someone who knows the secret key.

Appeared in State of the Art and Evolution of Computer Security and Industrial Cryptography, Lecture Notes in Computer Science 1528, Springer-Verlag, pp. 50-105. (C)1998 Springer-Verlag 
All the CBC-MAC variants are vulnerable to the birthday forgery attack (cf. Sect. 8.2), which requires a single chosen message and about $2^{r / 2}$ known messages (if $m=r$ ); if $m=r / 2$, an additional $2^{r / 2}$ chosen messages are required. For DES with $r=64,2^{32}$ texts are required. Note that with a fast software DES implementation, this number of texts can be collected in a few hours. Bellare et al. provide a proof of security for CBC-MAC [14] i.e., they establish a lower bound to break the system under certain assumptions on the block cipher. It almost matches the upper bound provided by the birthday forgery attack.

In 1995, Bellare et al. have proposed XOR-MAC [13]. It is a randomized algorithm and its security can again be reduced to that of the block cipher. It has the advantage that it is parallelizable and that it is incremental, i.e., small modifications to the message (and to the MAC) can be made at very low cost. The use of random bits clearly helps to improve security, but it has a cost in practical implementations. Also, the performance is typically 25 to $50 \%$ slower than CBC-MAC.

As has been pointed out earlier (cf. Sect. 4.4), if both authenticity and secrecy are protected using the same block cipher, the keys for both operations have to be different $[95,121,134]$.

\subsection{MAC Algorithms Based on a Hash Function}

The availability of fast dedicated hash functions (mainly of the MDx-family) has prompted several proposals for MAC algorithms based on these functions. The first proposals are the secret prefix and secret suffix methods which can be described as follows: $h_{K}(X)=h(K \| X), h_{K}(X)=h(X \| K)$. However, both have certificational weaknesses: the first one allows for extension attacks, and the second one opens the possibility of off-line attacks [140].

Another proposal is the secret envelope method, which can be described as $h_{K}(x)=h\left(K_{1}\|x\| K_{2}\right)$ (for example Internet RFC 1828, considered for IPSEC). Bellare et al. provide a security proof for this construction based on the assumption that the compression function of the hash function is pseudo-random [11]. While this is an interesting result, it should be pointed out that the compression function of most hash functions has not been evaluated with respect to this property. Preneel and van Oorschot have developed a key recovery attack on the envelope method that requires about $2^{n / 2}$ known texts [141] (cf. Sect. 8.2).

MDx-MAC, put forward by Preneel and van Oorschot in [140], extends the envelope method by also introducing secret key material into every iteration. This makes the pseudo-randomness assumption more plausible. Moreover, it precludes the key recovery attack by extending the keys to complete blocks.

HMAC, proposed by Bellare et al. [10], is currently the most popular construction in this class. It uses a nested construction (also with padded keys):

$$
h_{K}(X)=h\left(K_{2} \| h\left(K_{1} \| X\right)\right) .
$$

HMAC will be used for providing message authentication in IPSEC (Internet Protocol security) and in the SSL 3.0 (Secure Sockets Layer). The security of

Appeared in State of the Art and Evolution of Computer Security and Industrial Cryptography, Lecture Notes in Computer Science 1528, Springer-Verlag, pp. 50-105. (C)1998 Springer-Verlag 
HMAC is guaranteed if the hash function is collision resistant for a secret value $H_{0}$, and if the compression function itself is a secure MAC for 1 block (with the secret key in the $H_{i}$ input and the message in the $X_{i}$ input). While these assumptions are weaker, we believe that the the latter one still requires further validation for existing hash functions.

\subsection{Custom Designed MAC Algorithms}

The Message Authentication Algorithm (MAA) was published in 1983 by Davies and Clayden in response to a request of the UK Bankers Automated Clearing Services (BACS) [45, 46]. In 1987 it became a part of the ISO 8731 banking standard [86]. The algorithm is software oriented and has a 32-bit result. Recently several undesirable properties of MAA have been exposed by Preneel et al. [139]. They offer no immediate threat to current applications, but it would be advisable to check for the known classes of weak keys [139], and to change the key frequently. Nevertheless, it is anticipated that MAA will be withdrawn from the revised version of ISO 8731.

Several custom designed MAC algorithms in use have not been published, such as the S.W.I.F.T. authenticator, and the Swedish algorithm Data Seal. Proprietary MAC algorithms that can process only short messages include Telepass 1, the DECT standard authentication algorithm, the COMP128 algorithm (which is used by certain GSM operators and was shown to be weak), and the Sky Videocrypt system of British Sky Broadcasting.

\section{Methods of Attack on MAC Algorithms}

This section summarizes the known methods of attack on MAC algorithms. Three types of attacks will be distinguished:

1. attacks independent of the algorithm,

2. attacks dependent on the chaining,

3. high level attacks.

A MAC requires a more complex taxonomy than an MDC: the proposed taxonomy is equivalent to that of Goldwasser et al. [78] for digital signature schemes. Depending on the information available to an attacker, the following types of attacks are distinguished:

Known text attack: an attacker is able to examine some plaintexts and their corresponding MAC.

Chosen text attack: an attacker is able to select a set of texts, and subsequently he will obtain a list of MACs corresponding to these texts.

Adaptive chosen text attack: this is the most general attack where an attacker will choose a text and immediately receive the corresponding MAC: the choice of a text can depend on the outcome of previous queries. In some settings it is also relevant for an attacker to ask for a verification whether a MAC corresponds to a specific text.

Appeared in State of the Art and Evolution of Computer Security and Industrial Cryptography, Lecture Notes in Computer Science 1528, Springer-Verlag, pp. 50-105. (C)1998 Springer-Verlag 
"Breaking" a MAC algorithm can have different meanings:

Total break: this means that an attacker can determine the secret key $K$.

Universal forgery: an attacker can find an algorithm that is functionally equivalent to the MAC evaluation algorithm.

Selective forgery: an attacker can determine the correct MAC for a particular text chosen a priori by him.

Existential forgery: an attacker can determine the MAC for at least one text. As he has no control over this text, it may be random or nonsensical.

The second requirement in Definition 3 can now be restated as follows: it should be "hard" to perform an existential forgery with an adaptive chosen text attack. Note that obtaining a MAC for a text from the owner of the secret key is not considered as a forgery. In practice, an adaptive chosen text may not always be feasible; moreover, forgeries typically need to have specific redundancy to be of any practical use. However, it is in general better to be conservative and to require that MAC algorithms (and digital signature schemes) resist against the strongest attacks possible; the only restrictions one should consider are the number of texts and the computational capabilities.

For the attacks above, one requires that the forgery is verifiable, which means that the attacker knows that the forged MAC is correct with probability close to 1.

\subsection{Attacks Independent of the Algorithm}

This class of attacks depends only on the size $m$ in bits of the MAC (it will be assumed that an output transformation $g$ is used) and on the size $k$ in bits of the secret key; it is independent of the specific details of the algorithm. It is assumed that the MAC algorithm approximates a random function: if this is not the case this class of attacks will be even more successful.

Random Attack. A straightforward attack on a MAC algorithm consists of choosing an arbitrary new message, and subsequently guessing the MAC. This can be done in two ways: either one guesses the MAC directly, with a success probability of $2^{-m}$, or one guesses the key, and then computes the MAC, with success probability $2^{-k}$. This is a non-verifiable attack: an attacker does not know a priori whether his guess was correct. The feasibility of the attack depends on the number of trials that can be performed and on the expected value of successful attack; both are strongly application dependent. If the number of trials can be limited, or if the expected value is limited, a value of $m=32$ is sufficient. However, for most applications it is recommended that the size of the $\mathrm{MAC}$ is at least 64 bits.

Exhaustive Key Search. This attack requires approximately $k / m$ known textMAC pairs for a given key; one attempts to determine the key by trying oneby-one all the keys. The expected number of trials is equal $2^{k-1}$. In contrast

Appeared in State of the Art and Evolution of Computer Security and Industrial Cryptography, Lecture Notes in Computer Science 1528, Springer-Verlag, pp. 50-105. (C)1998 Springer-Verlag 
to a random attack, this attack is carried out off-line and it yields a complete break of the MAC. Contrary to what is believed, the size $k$ of the key does not only depend depends on the time span during which the MAC algorithm has to remain secure. One can argue that it should also depend on the time during which the MAC application (with the same key size) is deployed. This is based on the fact that just a single success during the lifetime of the system might be sufficient for an important fraud. Therefore, at present, 56 bits seems to be marginal; $75 \ldots 80$ bits is recommended as a reasonable value that provides some future protection (see also Sect. 6.1).

Birthday Attack. A birthday attack on a MAC algorithm might be possible, provided the opponent obtains a sufficient number of text-MAC pairs with a known or chosen text attack. In a typical application, this does not lead to a vulnerability. However, in certain special setting such as multi-destination secure electronic mail [123], a MAC algorithm should be second preimage resistant or even collision resistant for someone who knows the secret key. The MAC becomes then equivalent to an MDC.

\subsection{Attacks Dependent on the Chaining}

This class of attacks depends on some high level properties of the compression function $f$. They were published by Preneel and van Oorschot [140,141]; some related results by Bellare et al. can be found in $[10,11]$.

Exor forgery. This type of forgery only works if the value of $H_{i}$ is computed as a function of $H_{i-1} \oplus X_{i}$ (as in CBC-MAC), and if no output transformation is present. This attack has been observed independently by several researchers. The easiest variant requires only a single known text. Assume that the input $X$ and its padded version $\bar{X}$ consist of a single block. Assume that one knows $h_{K}(X)$; it follows immediately that $h_{K}\left(\bar{X} \|\left(X \oplus h_{K}(X)\right)\right)=h_{K}(X)$. This implies that one can construct a new message with the same MAC value, which is a forgery. Note that this attack applies even if a MAC algorithm key is used only once. A second variant requires two known texts. If one knows $h_{K}(X)$ and $h_{K}\left(X^{\prime}\right)$, a similar calculation shows that $h_{K}\left(\bar{X} \|\left(X^{\prime} \oplus h_{K}(X)\right)\right)=h_{K}\left(X^{\prime}\right)$. A third variant uses three known texts. If one knows $h_{K}(X), h_{K}(X \| Y)$, and $h_{K}\left(X^{\prime}\right)$, one knows that $h_{K}\left(X^{\prime} \| Y^{\prime}\right)=h_{K}(X \| Y)$ if $Y^{\prime}=Y \oplus h_{K}(X) \oplus h_{K}\left(X^{\prime}\right)$ (if $X$ and $Y$ fall on block boundaries). This also allows for a forgery, as an adversary can forge the MAC on $X^{\prime} \| Y^{\prime}$ given knowledge of the MACs for two known data strings and one chosen data string. Note that the above forgeries are on data strings of a specific form, which may not be a concern in all applications.

Some further thought shows that this attack cannot be precluded by appending the length. A strong output transformation does help. However, Knudsen has shown that if the output transformation consists of retaining the $m<r$ leftmost bits, a variant of the exor forgery attack applies; it requires $2^{(r-m) / 2}$ chosen texts [100].

Appeared in State of the Art and Evolution of Computer Security and Industrial Cryptography, Lecture Notes in Computer Science 1528, Springer-Verlag, pp. 50-105. (c)1998 Springer-Verlag 
Internal Collision Based Forgery. This section describes a generic forgery attack that applies against all iterated MAC algorithms (this includes all algorithms described in Sect. 7). The observation behind this attack is that if one can find a collision for the chaining variables, this can be used to construct a MAC forgery based on a single chosen text.

Let $\left(X, X^{\prime}\right)$ be a pair of message inputs with $h(X)=g\left(H_{t}\right)$ and $h\left(X^{\prime}\right)=$ $g\left(H_{t}^{\prime}\right)$. A chaining variable collision is said to occur when for some $i \leq t, H_{i}=H_{i}^{\prime}$, i.e., the intermediate chaining values coincide. An internal collision is said to occur when a chaining variable collision results in the situation where $H_{t}=H_{t}^{\prime}$. In the following it will be assumed that $g$ is deterministic: an internal collision yields a MAC collision. If $H_{t} \neq H_{t}^{\prime}$ but $g\left(H_{t}\right)=g\left(H_{t}^{\prime}\right)$, then an external collision is said to have occurred.

Lemma 1. An internal collision for an iterated MAC algorithm can be used to obtain a verifiable MAC forgery with a chosen text attack requiring only one requested MAC.

Proof. For an internal collision $\left(X, X^{\prime}\right)$, note that $h(X \| Y)=h\left(X^{\prime} \| Y\right)$. for any single block $Y$. Thus requesting a MAC for the single chosen text $X \| Y$, permits forgery - the MAC for $X^{\prime} \| Y$ is the same.

Note that the attack can be precluded by making the output transformation $g$ different for each MAC calculation, e.g., by including a sequence number or a sufficiently large random number in the calculation of $g$.

The following theorem indicates how one can identify an internal collision for the attack of Lemma 1.

Theorem 3 (Preneel-van Oorschot [140]). Let $h$ be an iterated MAC algorithm with $n$-bit chaining variable and $m$-bit result. An internal collision for $h$ can be found using u known text-MAC pairs and $v$ chosen texts. The expected values for $u$ and $v$ are as follows: $u=\sqrt{2} \cdot 2^{n / 2}$ and $v=0$ if the output transformation $g$ is a permutation; otherwise, $v$ is approximately

$$
2\left(2^{n-m}+\left\lfloor\frac{n-m}{m-1}\right\rfloor+1\right) .
$$

The two most important cases in practice are $n=m$ and $n=2 m$. In the first case, Theorem 3 indicates that only 4 chosen texts are required; if $n=2 m$, about $2^{m+1}$ chosen texts are required. Further optimizations of this attack are possible if the set of text-MAC pairs has a common sequence of $s$ trailing blocks. It should also be noted that the attack cannot be precluded by prepending the length of the input before the MAC calculation or by fixing the length of the input. The attack can be precluded by making the output transformation nondeterministic: this implies that chaining variable collisions will only lead with negligible probability to internal collisions.

Internal Collision Based Key Recovery. For some compression functions $f$, one can extend the internal collision attack to a key recovery attack [141].

Appeared in State of the Art and Evolution of Computer Security and Industrial Cryptography, Lecture Notes in Computer Science 1528, Springer-Verlag, pp. 50-105. (C)1998 Springer-Verlag 
The idea is to identify one or more internal collisions; for example, if $f$ is not a permutation for fixed $H_{i}$, an internal collision after the first message block gives the equation $f_{K}\left(X_{1}, H_{0}\right)=f_{K}\left(X_{1}^{\prime}, H_{0}\right)$, in which $K$ and possibly $H_{0}$ are unknown (it is assumed that the $H_{0}=I V$ is key dependent). For some compression functions $f$, one can obtain information on the secret key based on such relations. Examples are the envelope method with two different keys (Sect. 7.2), MAA, and COMP128 (Sect. 7.3). A related more subtle attack on RFC 1828 recovers the secret key in the output transformation (Sect. 7.2).

\subsection{High Level Attacks}

Even if the above attacks would not be feasible, special care has to be taken to avoid replay of messages and construction of valid messages by cutting and splicing others. Also the timeliness and the order of messages can be important. Attacks on this 'higher' level can be precluded by adding time stamps, serial numbers, or random challenges and through the use of sound cryptographic protocols. In the case of stored information, a restore can be avoided through the use of version numbers and the order of the blocks can be protected through adding the memory address to the information before the computation of the MAC.

\section{Digital Signature Schemes}

This section discusses some practical constructions for digital signatures. The most popular constructions are based on public-key techniques, but one can also construct digital signatures based on physical assumptions and based on conventional one-way functions.

\subsection{Based on Physical Assumptions}

A device like a smart card is called tamper resistant if one believes that it is difficult to access the secret key stored in it. One can use a smart card with a conventional cryptographic algorithm to build a signature scheme as follows: the 'signer' has a smart card that can only encipher with a secret key $K$, and each 'verifier' has a smart card that can only decipher with a secret key $K$. Forging a signature is hard if one believes that the devices are tamper resistant. The disadvantage of this approach is that the secret key $K$ has to be installed and stored securely within the smart card of both signer and verifier(s). This should be contrasted with the schemes of Sect. 9.3 where only the secret key of the signer is protected in a smart card.

\subsection{Based on a Conventional One-Way Function}

The first digital signature scheme based on a conventional one-way function (denoted with $g$ ) is the Diffie-Lamport one time signature [50]. In order to sign

Appeared in State of the Art and Evolution of Computer Security and Industrial Cryptography, Lecture Notes in Computer Science 1528, Springer-Verlag, pp. 50-105. (C)1998 Springer-Verlag 
a single bit message, the sender randomly selects a pair $x_{1}, x_{2} \in \operatorname{Dom}(g)$ and computes $y_{1}=g\left(x_{1}\right)$ and $y_{2}=g\left(x_{2}\right)$. Next he puts $y_{1}, y_{2}$ in an authenticated public file. If he wants to sign the message, he reveals $x_{1}$ if the message bit equals 0 and $x_{2}$ if the message bit equals 1 . Subsequently the receiver can verify that $y_{i}=g\left(x_{i}\right)$. A second well known construction is the Rabin scheme [146]; the 'cut and choose' technique proves to be a very powerful building block for other cryptographic protocols.

The main disadvantage of these schemes are the size of keys and signatures and the fact that they can only be used for a fixed number of signatures (often only once). The optimizations by Merkle [117] yield a scheme that is almost practical; Bleichenbacher and Maurer provide a generalization of these methods [21]. These basic schemes have served as a starting point for complexity theoretic constructions.

\subsection{Based on Public-Key Techniques}

An elegant solution to construct digital signatures was proposed by Diffie and Hellman in their seminal 1976 paper [50] in which they introduced the concept of trapdoor one-way functions. These functions are easy to compute in one direction and difficult to compute in the other direction, except for someone who knows the 'trapdoor' information. Information can then be digitally signed if the sender transforms the information with his secret key (the trapdoor information). The receiver can then verify the digital signature by applying the transformation in the 'easy direction' using the public key. The signature is unique to the sender because she is the only one who possesses the secret information.

Trapdoor one-way functions can also be used for public-key encryption systems, where the receiver can make his key public through an integrity protected channel. The encryption operation is then the transformation with the public key, and the decryption the inverse transformation (using the secret key or trapdoor). Note that in general the signing operation and the verification operation of a signature scheme are not equivalent to decryption and encryption respectively; this widespread misunderstanding has been created because this equivalence holds for RSA, which is discussed in the next paragraph.

The first 'secure' trapdoor one-way permutation was proposed by Rivest, Shamir, and Adleman in 1977 [153]. The RSA public-key cryptosystem is based on modular exponentiation and its security relies on the difficulty of extracting modular roots modulo an integer that is the product of two large primes (this problem is related to factoring such integers). It is widely used and has become a "de facto" standard. A variant of RSA that is provably equivalent to factoring was proposed by Rabin [147]; it is based on modular squaring. The RSA and the Rabin scheme are the only public-key signature schemes that have a deterministic signing procedure, i.e., the signing process does not need any random bits. Other efficient variants have been proposed based on the fact that even finding a good approximation for a modular square root is hard if the factorization of the modulus is not known. An example in this class is ESIGN [68]; this scheme is based on a modulus of the form $n=p^{2} q$.

Appeared in State of the Art and Evolution of Computer Security and Industrial Cryptography, Lecture Notes in Computer Science 1528, Springer-Verlag, pp. 50-105. (C)1998 Springer-Verlag 
A second class of schemes is based on the discrete logarithm problem. The first scheme in this class was suggested by ElGamal in 1984 [57]. Bleichenbacher points out some vulnerabilities of this scheme in [20]. An optimized variant was put forward by Agnew et al. [2]. The Digital Signature Algorithm standardized by NIST [66] also belongs to this class. It is based on the difficulty of the discrete logarithm in $G F(p)$, where $p$ is a large prime number (512 to 1024 bits) such that $p-1$ has a 160 -bit prime divisor $q$. The scheme exploits this structure to optimize storage and computation as suggested by Schnorr [158] for a different scheme. The advantage of schemes based on the discrete logarithm is that they can be implemented in any group for which the discrete logarithm is believed to be hard. Recently some promising results have been achieved with schemes based on elliptic curves over finite fields (e.g., [49, 114]).

A third class of schemes is derived from zero-knowledge identification protocols; the schemes can again be divided into two classes according to the underlying assumption. The variants based on factoring are Guillou-Quisquater [145] and Ohta-Okamoto [131], and a variant with exponent 2 is the Feige-FiatShamir scheme [60]. The variants based on the discrete logarithm problem are the schemes by Schnorr [158] and Okamoto [130].

The schemes that have been mentioned in this section are all reasonably efficient, but they do not have the same level of provable security, i.e., the relation with the underlying hard problem might be different. What is important is how tight the relation is between forging a signature and breaking the underlying hard problem (for example extracting random modular roots for RSA). The schemes for which an even stronger reduction is possible (e.g., [23,78]) or the schemes based on more general assumptions (e.g., [128, 156]) are however less practical. The closest to practical is the construction by Dwork and Naor [56], which requires between two and six RSA computations and some extra public storage; signatures are also six times longer than the modulus.

\subsection{Combining a Hash Function and a Signature Scheme}

The main idea to speed up all digital signature schemes is to compress the information to be signed with an MDC to a string of fixed length (sometimes called "imprint") and to sign this string. In order to verify the signature, the hash result is recalculated and is input to the computations of the verification procedure. For digital signatures schemes with message recovery, such as RSA, the imprint is recovered during the verification procedure and compared to the hash result of the received message (e.g., ISO/IEC 97976-1 [87]). There also exists a mixed mode, for which in addition to the imprint, part of the message is recovered (ISO/IEC 97976-2 [87]).

The advantages of combining a digital signature scheme with a hash function are the following:

1. The size of the signature can be reduced from the size of the information to one block length, independent of the length of the signed information; for ISO/IEC 97976-2, the overhead of an RSA signature is reduced to approximately 23 bytes.

Appeared in State of the Art and Evolution of Computer Security and Industrial Cryptography, Lecture Notes in Computer Science 1528, Springer-Verlag, pp. 50-105. (C)1998 Springer-Verlag 
2. The sign and verify function of most known signature schemes are several orders of magnitude slower in hardware and software than MDCs.

3. If information is signed that is longer than one block, it is easy to manipulate these individual blocks. The simplest example is a reordering of blocks.

4. The algebraic structure of the message space can be destroyed. In the case of RSA [58] and discrete logarithm based signatures, the message space has a multiplicative structure, i.e., the signature of the product of two messages equals the product of their signatures. Examples of how this algebraic structure can be exploited in a protocol are described by Gordon [79] and Moore [127].

5. The reblocking problem can be avoided. This problem occurs when both privacy and authentication of a short message are protected (for example, using RSA), and the result of the signature process is larger than the modulus used for encryption.

6. The signature protocol will not be useful for an opponent trying to obtain the plaintext corresponding to encrypted messages. This can only happen if one uses the same public-key cryptosystem and keys for privacy protection and authentication, which is not good practice anyway.

For the zero-knowledge based signature schemes, the hash function is an integral part of the signing and verification operations, as it links together the information to be signed and some unpredictable and signature dependent information. However, this does not imply that it is sufficient that the hash function is a OWHF, as one always has to take into consideration a cheating signer. Moreover, a somewhat stronger 'randomization' property is required in addition to collision resistance. Note that the requirements are different in the case of entity authentication; Girault and Stern have pointed out that resistance to multiple collisions (together with some 'randomization' properties) is sufficient [76].

In some cases there can be an unfortunate interaction between the digital signature scheme and the hash function (cf. Sect. 6.3). To avoid this problem, one can impose additional conditions on the hash function (e.g., it should be nonhomomorphic, correlation free $[130], \ldots)$. An alternative approach, which seems preferable to us, is to solve the problems with the signature scheme: an example is the redundancy scheme for RSA (and its extension to even exponents) which was standardized in [87]: it destroys the homomorphic property as explained by Guillou et al. [80]. However, this is not sufficient to reduce the security of the signature scheme to that of extracting random modular roots. This has been achieved by PSS or provably secure signing as proposed by Bellare and Rogaway [16]; they prove the security of PSS in the random oracle model; this means that the hash function is expected to behave in an 'ideal' way.

\subsection{Selecting a Signature Scheme}

When selecting a particular signature scheme, one has to consider the following aspects:

Appeared in State of the Art and Evolution of Computer Security and Industrial Cryptography, Lecture Notes in Computer Science 1528, Springer-Verlag, pp. 50-105. (C)1998 Springer-Verlag 
efficiency in terms of storage and computation: number of operations for signing and verifying, size of secret and public key, size of the signature, and the possibility of preprocessing.

random: randomized signatures will probably leak less information about the secret key, but on the other hand the signer needs to produce random bits in a secure way (if the random input is revealed, not only the signature but the secret key might be compromised).

security: is the scheme well established, and how strong is the reduction to the underlying hard problem?

hash function: the requirements to be imposed on the hash function.

patents and standards: these non-technical aspects will also influence the choice of a particular scheme.

\subsection{Some Practical Considerations}

After a slow start, digital signature schemes are now getting more widely accepted. An important problem is a user who looses his secret key, or who claims he has lost his secret key. In both cases he will try to revoke his digital signatures. Any practical signature scheme has to implement procedures for the revocation of keys. In order to minimize this risk, one can store the secret key in a tamper resistant device (e.g., a smart card), such that even the owner does not know it, and one can maintain black lists for users with a bad history. This will make fraudulent revocations very difficult. A second problem is that after some time, the signature scheme might be broken (due to advances in cryptanalysis); in order to avoid that the legal validity of the signature (more precisely, of the contract created with the signature) is lost, one needs to register a digital signature, or to use arbitrated signature schemes, where a third party is involved in the signing process. Recently significant progress have been made towards legal acceptance of digital signatures under several jurisdictions (for example, Germany, Utah, and the European Directive), but progress is still rather slow. Legal support of digital signatures is certainly necessary to get a wide acceptance and will be a significant step forward to the wide deployment of electronic commerce.

\section{Conclusions}

For protecting information authentication, several cryptographic techniques are available: MACs, MDCs, and digital signatures.

For MACs, efficient standards exist that are widely accepted. The security level of the widely used CBC-MAC is lower than anticipated, but as soon as 128bit block ciphers will be available, this problem will have been resolved. A recent trend is to construct MAC algorithms based on MDCs. For concrete candidates, some additional research is required to assess the security of these proposals. For

Appeared in State of the Art and Evolution of Computer Security and Industrial Cryptography, Lecture Notes in Computer Science 1528, Springer-Verlag, pp. 50-105. (C)1998 Springer-Verlag 
applications where performance and communication overhead are crucial (such as authentication of packets at the IP level) MAC algorithms are still preferred over digital signatures.

For authentication between mutually distrusting parties, as is required in electronic commerce, digital signatures offer an essential solution. In the coming years, one can expect a wide deployment of the infrastructure to support this technology, the so-called PKIs (public key infrastructures). Further theoretical developments together with legal support will make digital signatures widely accepted.

The most important application of MDCs is in digital signatures, but they have been used as a flexible tool in different cryptographic constructions. While a few MDCs seems to be widely trusted, further research is required to increase our understanding of design principles and to provide more efficient constructions.

\section{References}

1. V. Afanassiev, C. Gehrmann, B. Smeets, "Fast message authentication using efficient polynomial evaluation," Fast Software Encryption, LNCS 1267, E. Biham, Ed., Springer-Verlag, 1997, pp. 190-204.

2. G.B. Agnew, R.C. Mullin, S.A. Vanstone, "Common application protocols and their security characteristics," CALMOS CA34C168 Application Notes, U.S. Patent Number 4,745,568, August 1989.

3. A.V. Aho, J.E. Hopcroft, J.D. Ullman, "The Design and Analysis of Computer Algorithms," Addison-Wesley, 1974.

4. W. Aiello, R. Venkatesan, "Foiling birthday attacks in length-doubling transformations. Benes: a non-reversible alternative to Feistel," Advances in Cryptology, Proceedings Eurocrypt'96, LNCS 1070, U. Maurer, Ed., Springer-Verlag, 1996, pp. 307-320.

5. W. Aiello, S. Haber, R. Venkatesan, "New constructions for secure hash functions," Fast Software Encryption, LNCS 1372, S. Vaudenay, Ed., Springer-Verlag, 1998, pp. 150-167.

6. M. Ajtai, "Generating hard instances of lattice problems," Proc. 28th ACM Symposium on the Theory of Computing, 1996, pp. 99-108.

7. R. Anderson, E. Biham, "Tiger: A new fast hash function," Fast Software Encryption, LNCS 1039, D. Gollmann, Ed., Springer-Verlag, 1996, pp. 89-97.

8. ANSI X9.9-1986 (Revised), "American National Standard for Financial Institution Message Authentication (Wholesale)," ANSI, New York.

9. ANSI X9.19 "Financial Institution Retail Message Authentication," American Bankers Association, August 13, 1986.

10. M. Bellare, R. Canetti, H. Krawczyk, "Keying hash functions for message authentication," Advances in Cryptology, Proceedings Crypto'96, LNCS 1109, N. Koblitz, Ed., Springer-Verlag, 1996, pp. 1-15.

Full version: http:// www.research.ibm.com/security/.

11. M. Bellare, R. Canetti, H. Krawczyk, "Pseudorandom functions revisited: The cascade construction and its concrete security," Proc. 37th Annual Symposium on the Foundations of Computer Science, IEEE, 1996, pp. 514-523.

Full version via http://www-cse.ucsd.edu/users/mihir.

Appeared in State of the Art and Evolution of Computer Security and Industrial Cryptography, Lecture Notes in Computer Science 1528, Springer-Verlag, pp. 50-105. (C)1998 Springer-Verlag 
12. M. Bellare, O. Goldreich, S. Goldwasser, "Incremental cryptography: the case of hashing and signing," Advances in Cryptology, Proceedings Crypto'94, LNCS 839, Y. Desmedt, Ed., Springer-Verlag, 1994, pp. 216-233.

13. M. Bellare, R. Guérin, P. Rogaway, "XOR MACs: new methods for message authentication using block ciphers," Advances in Cryptology, Proceedings Crypto'95, LNCS 963, D. Coppersmith, Ed., Springer-Verlag, 1995, pp. 15-28.

14. M. Bellare, J. Kilian, P. Rogaway, "The security of cipher block chaining," Advances in Cryptology, Proceedings Crypto'94, LNCS 839, Y. Desmedt, Ed., Springer-Verlag, 1994, pp. 341-358.

15. M. Bellare, D. Micciancio, "A new paradigm for collision-free hashing: incrementality at reduced cost," Advances in Cryptology, Proceedings Eurocrypt'97, LNCS 1233, W. Fumy, Ed., Springer-Verlag, 1997, pp. 163-192.

16. M. Bellare, P. Rogaway, "The exact security of digital signatures - how to sign with RSA and Rabin," Advances in Cryptology, Proceedings Eurocrypt'96, LNCS 1070, U. Maurer, Ed., Springer-Verlag, 1996, pp. 399-416.

17. M. Bellare, P. Rogaway, "Collision-resistant hashing: towards making UOWHFs practical," Advances in Cryptology, Proceedings Crypto'97, LNCS 1294, B. Kaliski, Ed., Springer-Verlag, 1997, pp. 470-484.

18. E. Biham, "On the applicability of differential cryptanalysis to hash functions," E.I.S.S. Workshop on Cryptographic Hash Functions, Oberwolfach (D), March 25-27, 1992.

19. E. Biham, A. Shamir, "Differential Cryptanalysis of the Data Encryption Standard," Springer-Verlag, 1993.

20. D. Bleichenbacher, "Generating ElGamal signatures without knowing the secret key," Advances in Cryptology, Proceedings Eurocrypt'96, LNCS 1070, U. Maurer, Ed., Springer-Verlag, 1996, pp. 10-18.

21. D. Bleichenbacher, U.M. Maurer, "Directed acyclic graphs, one-way functions and digital signatures," Advances in Cryptology, Proceedings Crypto'94, LNCS 839, Y. Desmedt, Ed., Springer-Verlag, 1994, pp. 75-82.

22. D. Boneh, M. Franklin, "Efficient generation of shared RSA keys," Advances in Cryptology, Proceedings Crypto'97, LNCS 1294, B. Kaliski, Ed., Springer-Verlag, 1997, pp. 425-439.

23. J. Bos, D. Chaum, "Provably unforgeable signatures," Advances in Cryptology, Proceedings Crypto'92, LNCS 740, E.F. Brickell, Ed., Springer-Verlag, 1993, pp. $1-14$.

24. B.O. Brachtl, D. Coppersmith, M.M. Hyden, S.M. Matyas, C.H. Meyer, J. Oseas, S. Pilpel, M. Schilling, "Data Authentication Using Modification Detection Codes Based on a Public One Way Encryption Function," U.S. Patent Number 4,908,861, March 13, 1990.

25. G. Brassard, "On computationally secure authentication tags requiring short secret shared keys," Advances in Cryptology, Proceedings Crypto'82, D. Chaum, R.L. Rivest, and A.T. Sherman, Eds., Plenum Press, New York, 1983, pp. 79-86.

26. P. Camion, J. Patarin, "The knapsack hash function proposed at Crypto' 89 can be broken," Advances in Cryptology, Proceedings Eurocrypt'91, LNCS 547, D.W. Davies, Ed., Springer-Verlag, 1991, pp. 39-53.

27. C.M. Campbell Jr., "Design and specification of cryptographic capabilities," D.K. Branstad, Ed., Computer Security and the Data Encryption Standard, NBS Special Publication 500-27, U.S. Department of Commerce, 1977, pp. 54-66.

28. J.L. Carter, M.N. Wegman, "Universal classes of hash functions," Journal of Computer and System Sciences, Vol. 18, 1979, pp. 143-154.

Appeared in State of the Art and Evolution of Computer Security and Industrial Cryptography, Lecture Notes in Computer Science 1528, Springer-Verlag, pp. 50-105. (C)1998 Springer-Verlag 
29. C.C.I.T.T. X.509, "The Directory - Authentication Framework," Recommendation, 1988, (same as ISO/IEC 9594-8, 1989).

30. F. Chabaud, A. Joux, "Differential collisions: an explanation for SHA-1," Advances in Cryptology, Proceedings Crypto'98, LNCS 1462, H. Krawczyk, Ed., Springer-Verlag, 1998, pp. 56-71.

31. C. Charnes, J. Pieprzyk, "Attacking the $S L_{2}$ hashing scheme," Advances in Cryptology, Proceedings Asiacrypt'94, LNCS 917, J. Pieprzyk and R. Safavi-Naini, Eds., Springer-Verlag, 1995, pp. 322-330.

32. D. Chaum, S. Roijakkers, "Unconditionally-secure digital signatures," Advances in Cryptology, Proceedings Crypto'90, LNCS 537, S. Vanstone, Ed., SpringerVerlag, 1991, pp. 206-214.

33. D. Chaum, E. van Heijst, B. Pfitzmann, "Cryptographically strong undeniable signatures, unconditionally secure for the signer," Advances in Cryptology, Proceedings Crypto'91, LNCS 576, J. Feigenbaum, Ed., Springer-Verlag, 1992, pp. 470484.

34. D. Coppersmith, "Another birthday attack," Advances in Cryptology, Proceedings Crypto'85, LNCS 218, H.C. Williams, Ed., Springer-Verlag, 1985, pp. 14-17.

35. D. Coppersmith, "Analysis of ISO/CCITT Document X.509 Annex D," IBM T.J. Watson Center, Yorktown Heights, N.Y., 10598, Internal Memo, June 11, 1989, (also ISO/IEC JTC1/SC20/WG2/N160).

36. D. Coppersmith, B. Preneel, "Comments on MASH-1 and MASH-2," February 21, 1995, ISO/IEC JTC1/SC27/N1055.

37. J. Daemen, "Cipher and Hash Function Design. Strategies Based on Linear and Differential Cryptanalysis," Doctoral Dissertation, Katholieke Universiteit Leuven, 1995.

38. J. Daemen, C. Clapp, "Fast hashing and stream encryption with PANAMA," Fast Software Encryption, LNCS 1372, S. Vaudenay, Ed., Springer-Verlag, 1998, pp. 60-74.

39. J. Daemen, R. Govaerts, J. Vandewalle, "A framework for the design of oneway hash functions including cryptanalysis of Damgård's one-way function based on a cellular automaton," Advances in Cryptology, Proceedings Asiacrypt'91, LNCS 739, H. Imai, R.L. Rivest, and T. Matsumoto, Eds., Springer-Verlag, 1993, pp. 82-96.

40. I.B. Damgård, "Collision free hash functions and public key signature schemes," Advances in Cryptology, Proceedings Eurocrypt'87, LNCS 304, D. Chaum and W.L. Price, Eds., Springer-Verlag, 1988, pp. 203-216.

41. I.B. Damgård, "The application of claw free functions in cryptography," $P h D$ Thesis, Aarhus University, Mathematical Institute, 1988.

42. I.B. Damgård, "A design principle for hash functions," Advances in Cryptology, Proceedings Crypto'89, LNCS 435, G. Brassard, Ed., Springer-Verlag, 1990, pp. $416-427$.

43. I.B. Damgård, T.P. Pedersen, B. Pfitzmann, "On the existence of statistically hiding bit commitment schemes and fail-stop signatures," Advances in Cryptology, Proceedings Crypto'93, LNCS 773, D. Stinson, Ed., Springer-Verlag, 1994, pp. $250-265$.

44. D. Davies, W. L. Price, "The application of digital signatures based on public key cryptosystems," NPL Report DNACS 39/80, December 1980.

45. D. Davies, "A message authenticator algorithm suitable for a mainframe computer," Advances in Cryptology, Proceedings Crypto'84, LNCS 196, G.R. Blakley and D. Chaum, Eds., Springer-Verlag, 1985, pp. 393-400.

Appeared in State of the Art and Evolution of Computer Security and Industrial Cryptography, Lecture Notes in Computer Science 1528, Springer-Verlag, pp. 50-105. (c)1998 Springer-Verlag 
46. D. Davies, W.L. Price, "Security for Computer Networks: an Introduction to Data Security in Teleprocessing and Electronic Funds Transfer (2nd edition)," Wiley \& Sons, 1989.

47. B. den Boer, A. Bosselaers, "An attack on the last two rounds of MD4," Advances in Cryptology, Proceedings Crypto'91, LNCS 576, J. Feigenbaum, Ed., SpringerVerlag, 1992, pp. 194-203.

48. B. den Boer, A. Bosselaers, "Collisions for the compression function of MD5," Advances in Cryptology, Proceedings Eurocrypt'93, LNCS 765, T. Helleseth, Ed., Springer-Verlag, 1994, pp. 293-304.

49. E. De Win, B. Preneel, "Elliptic curve public-key cryptosystems - an introduction," This Volume.

50. W. Diffie, M.E. Hellman, "New directions in cryptography," IEEE Trans. on Information Theory, Vol. IT-22, No. 6, 1976, pp. 644-654.

51. H. Dobbertin, "RIPEMD with two-round compress function is not collisionfree," Journal of Cryptology, Vol. 10, No. 1, 1997, pp. 51-69.

52. H. Dobbertin, "Cryptanalysis of MD4," Journal of Cryptology, Vol. 11, No. 4, 1998, pp. 253-271. See also Fast Software Encryption, LNCS 1039, D. Gollmann, Ed., Springer-Verlag, 1996, pp. 53-69.

53. H. Dobbertin, "The status of MD5 after a recent attack," CryptoBytes, Vol. 2, No. 2, Summer 1996, pp. 1-6.

54. H. Dobbertin, "The first two rounds of MD4 are not one-way," Fast Software Encryption, LNCS 1372, S. Vaudenay, Ed., Springer-Verlag, 1998, pp. 284-292.

55. H. Dobbertin, A. Bosselaers, B. Preneel, "RIPEMD-160: a strengthened version of RIPEMD," Fast Software Encryption, LNCS 1039, D. Gollmann, Ed., SpringerVerlag, 1996, pp. 71-82.

See also http://www.esat.kuleuven.ac.be/ bosselae/ripemd160.

56. C. Dwork, M. Naor, "An efficient existentially unforgeable signature scheme and its applications," Advances in Cryptology, Proceedings Crypto'94, LNCS 839, Y. Desmedt, Ed., Springer-Verlag, 1994, pp. 234-246.

57. T. ElGamal, "A public key cryptosystem and a signature scheme based on discrete logarithms," IEEE Trans. on Information Theory, Vol. IT-31, No. 4, 1985, pp. 469-472.

58. J.H. Evertse, E. Van Heijst, "Which new RSA-signatures can be computed from certain given RSA-signatures?" Journal of Cryptology, Vol. 5, No. 1, 1992, pp. 4152.

59. V. Fåk, "Repeated uses of codes which detect deception," IEEE Trans. on Information Theory, Vol. IT-25, No. 2, 1979, pp. 233-234.

60. U. Feige, A. Fiat, A. Shamir, "Zero knowledge proofs of identity," Journal of Cryptology, Vol. 1, No. 2, 1988, pp. 77-94.

61. FIPS 46, "Data Encryption Standard," Federal Information Processing Standard, National Bureau of Standards, U.S. Department of Commerce, Washington D.C., January 1977 (revised as FIPS 46-1:1988; FIPS 46-2:1993).

62. FIPS 81, "DES Modes of Operation," Federal Information Processing Standard, National Bureau of Standards, US Department of Commerce, Washington D.C., December 1980.

63. FIPS 113, "Computer Data Authentication," Federal Information Processing Standard, National Bureau of Standards, US Department of Commerce, Washington D.C., May 1985.

64. FIPS 180, "Secure Hash Standard," Federal Information Processing Standard (FIPS), Publication 180, National Institute of Standards and Technology, US Department of Commerce, Washington D.C., May 11, 1993.

Appeared in State of the Art and Evolution of Computer Security and Industrial Cryptography, Lecture Notes in Computer Science 1528, Springer-Verlag, pp. 50-105. (C)1998 Springer-Verlag 
65. FIPS 180-1, "Secure Hash Standard," Federal Information Processing Standard (FIPS), Publication 180-1, National Institute of Standards and Technology, US Department of Commerce, Washington D.C., April 17, 1995.

66. FIPS 186, "Digital Signature Standard," Federal Information Processing Standard (FIPS), Publication 186, National Institute of Standards and Technology, US Department of Commerce, Washington D.C., May 19, 1994.

67. Y. Frankel, P. D. MacKenzie, M. Yung, "Robust efficient distributed RSA-key generation," Proc. 30th ACM Symposium on the Theory of Computing, 1998.

68. A. Fujioka, T. Okamoto, S. Miyaguchi, "ESIGN: an efficient digital signature implementation for smart cards," Advances in Cryptology, Proceedings Eurocrypt'91, LNCS 547, D.W. Davies, Ed., Springer-Verlag, 1991, pp. 446-457.

69. W. Geiselmann, "A note on the hash function of Tillich and Zémor," Cryptography and Coding. 5th IMA Conference, C. Boyd, Ed., Springer-Verlag, 1995, pp. 257263.

70. J.K. Gibson, "Some comments on Damgård's hashing principle," Electronic Letters, Vol. 26, No. 15, 1990, pp. 1178-1179.

71. J.K. Gibson, "Discrete logarithm hash function that is collision free and one way," IEE Proceedings-E, Vol. 138, No. 6, November 1991, pp. 407-410.

72. E. Gilbert, F. MacWilliams, N. Sloane, "Codes which detect deception," Bell System Technical Journal, Vol. 53, No. 3, 1974, pp. 405-424.

73. M. Girault, "Hash-functions using modulo-n operations," Advances in Cryptology, Proceedings Eurocrypt'87, LNCS 304, D. Chaum and W.L. Price, Eds., Springer-Verlag, 1988, pp. 217-226.

74. M. Girault, R. Cohen, M. Campana, "A generalized birthday attack," Advances in Cryptology, Proceedings Eurocrypt'88, LNCS 330, C.G. Günther, Ed., SpringerVerlag, 1988, pp. 129-156.

75. M. Girault, J.-F. Misarsky, "Selective forgery of RSA signatures using redundancy," Advances in Cryptology, Proceedings Eurocrypt'97, LNCS 1233, W. Fumy, Ed., Springer-Verlag, 1997, pp. 495-507.

76. M. Girault, J. Stern, "On the length of cryptographic hash-values used in identification schemes," Advances in Cryptology, Proceedings Crypto'94, LNCS 839, Y. Desmedt, Ed., Springer-Verlag, 1994, pp. 202-215.

77. O. Goldreich, S. Goldwasser, S. Halevi, "Collision-free hashing from lattice problems," Theory of Cryptography Library, http://philby.ucsd.edu/cryptolib.html, 96-09, July 1996.

78. S. Goldwasser, S. Micali, R.L. Rivest, "A digital signature scheme secure against adaptive chosen-message attacks," SIAM Journal on Computing, Vol. 17, No. 2, 1988, pp. 281-308.

79. J.A. Gordon, "How to forge RSA certificates," Electronics Letters, Vol. 21, No. 9, 1985, pp. 377-379.

80. L.C. Guillou, J.-J. Quisquater, M. Walker, P. Landrock, C. Shaer, "Precautions taken against various potential attacks in ISO/IEC DIS 9796," Advances in Cryptology, Proceedings Eurocrypt'90, LNCS 473, I.B. Damgård, Ed., SpringerVerlag, 1991, pp. 465-473.

81. S. Halevi, H. Krawczyk, "MMH: Software message authentication in the Gbit/second rates," Fast Software Encryption, LNCS 1267, E. Biham, Ed., Springer-Verlag, 1997, pp. 172-189.

82. M. Hellman, R. Merkle, R. Schroeppel, L. Washington, W. Diffie, S. Pohlig, P. Schweitzer, "Results of an initial attempt to cryptanalyze the NBS Data Encryption Standard," Information Systems Lab., Dept. of Electrical Eng., Stanford Univ., 1976.

Appeared in State of the Art and Evolution of Computer Security and Industrial Cryptography, Lecture Notes in Computer Science 1528, Springer-Verlag, pp. 50-105. (C)1998 Springer-Verlag 
83. W. Hohl, X. Lai, T. Meier, C. Waldvogel, "Security of iterated hash functions based on block ciphers," Advances in Cryptology, Proceedings Crypto'93, LNCS 773, D. Stinson, Ed., Springer-Verlag, 1994, pp. 379-390.

84. R. Impagliazzo, M. Naor, "Efficient cryptographic schemes provably as secure as subset sum," Journal of Cryptology, Vol. 9, No. 4, 1996, pp. 199-216.

85. ISO 7498-2, "Information processing - Open systems interconnection - Basic reference model - Part 2: Security architecture," 1987.

86. ISO 8731, "Banking - approved algorithms for message authentication - Part 1: DEA," 1987. "Part 2, Message Authentication Algorithm (MAA)," 1987.

87. ISO/IEC 9796, "Information technology - Security techniques - Part 1: Digital signature scheme giving message recovery," 1991, "Part 2: Mechanisms using a hash-function," 1997.

88. ISO/IEC 9797, "Information technology - Data cryptographic techniques - Data integrity mechanisms using a cryptographic check function employing a block cipher algorithm," ISO/IEC, 1994.

89. ISO/IEC 10116, "Information technology - Security techniques - Modes of operation of an n-bit block cipher algorithm," 1996.

90. ISO/IEC 10118, "Information technology - Security techniques - Hash-functions, Part 1: General", 1994, "Part 2: Hash-functions using an n-bit block cipher algorithm," 1994, "Part 3: Dedicated hash-functions," 1998. "Part 4: Hash-functions using modular arithmetic," (FDIS) 1998.

91. "Hash functions using a pseudo random algorithm," ISO-IEC/JTC1/SC27/WG2 N98, Japanese contribution, 1991.

92. M. Jakubowski, R. Venkatesan, "The chain \& sum primitive and its applications to MACs and stream ciphers," Advances in Cryptology, Proceedings Eurocrypt'98, LNCS 1403, K. Nyberg, Ed., Springer-Verlag, 1998, pp. 281-293.

93. T. Johansson, "Bucket hashing with a small key size," Advances in Cryptology, Proceedings Eurocrypt'97, LNCS 1233, W. Fumy, Ed., Springer-Verlag, 1997, pp. $149-162$.

94. A. Joux, L. Granboulan, "A practical attack against knapsack based hash functions," Advances in Cryptology, Proceedings Eurocrypt'94, LNCS 950, A. De Santis, Ed., Springer-Verlag, 1995, pp. 58-66.

95. R.R. Jueneman, S.M. Matyas, C.H. Meyer, "Message authentication with Manipulation Detection Codes," Proc. 1983 IEEE Symposium on Security and Privacy, 1984 , pp. $33-54$.

96. R.R. Jueneman, "A high speed Manipulation Detection Code," Advances in Cryptology, Proceedings Crypto'86, LNCS 263, A.M. Odlyzko, Ed., SpringerVerlag, 1987, pp. 327-347.

97. G.A. Kabatianskii, T. Johansson, B. Smeets, "On the cardinality of systematic A-codes via error correcting codes," IEEE Trans. on Information Theory, Vol. IT42, No. 2, 1996, pp. 566-578.

98. B.S. Kaliski, "The MD2 Message-Digest algorithm," Request for Comments (RFC) 1319, Internet Activities Board, Internet Privacy Task Force, April 1992.

99. L.R. Knudsen, "New potentially 'weak' keys for DES and LOKI," Advances in Cryptology, Proceedings Eurocrypt'94, LNCS 950, A. De Santis, Ed., SpringerVerlag, 1995, pp. 419-424.

100. L. Knudsen, "Chosen-text attack on CBC-MAC," Electronics Letters, Vol. 33, No. 1, 1997, pp. 48-49.

101. L.R. Knudsen, X. Lai, B. Preneel, "Attacks on fast double block length hash functions," Journal of Cryptology, Vol. 11, No. 1, Winter 1998, pp. 59-72.

Appeared in State of the Art and Evolution of Computer Security and Industrial Cryptography, Lecture Notes in Computer Science 1528, Springer-Verlag, pp. 50-105. (c)1998 Springer-Verlag 
102. L.R. Knudsen, B. Preneel, "Fast and secure hashing based on codes," Advances in Cryptology, Proceedings Crypto'97, LNCS 1294, B. Kaliski, Ed., Springer-Verlag, 1997, pp. $485-498$.

103. L. Knudsen, B. Preneel, "MacDES: MAC algorithm based on DES," Electronics Letters, Vol. 34, No. 9, 1998, pp. 871-873,

104. H. Krawczyk, "LFSR-based hashing and authentication," Advances in Cryptology, Proceedings Crypto'94, LNCS 839, Y. Desmedt, Ed., Springer-Verlag, 1994, pp. $129-139$.

105. H. Krawczyk, "New hash functions for message authentication," Advances in Cryptology, Proceedings Eurocrypt'95, LNCS 921, L.C. Guillou and J.J. Quisquater, Eds., Springer-Verlag, 1995, pp. 301-310.

106. X. Lai, "On the Design and Security of Block Ciphers," ETH Series in Information Processing, Vol. 1, J. Massey, Ed., Hartung-Gorre Verlag, Konstanz, 1992.

107. X. Lai, J.L. Massey, "Hash functions based on block ciphers," Advances in Cryptology, Proceedings Eurocrypt'92, LNCS 658, R.A. Rueppel, Ed., Springer-Verlag, 1993, pp. 55-70.

108. A. Lenstra, H. Lenstra, L. Lovász, "Factoring polynomials with rational coefficients," Mathematischen Annalen, Vol. 261, pp. 515-534, 1982.

109. M. Matsui, "The first experimental cryptanalysis of the Data Encryption Standard," Advances in Cryptology, Proceedings Crypto'94, LNCS 839, Y. Desmedt, Ed., Springer-Verlag, 1994, pp. 1-11.

110. J.L. Massey, "Cryptography - A selective survey," Digital Communications (Proc. 1985 International Tirrenia Workshop), E. Biglieri, G. Prati, Eds., Elsevier Science Publ., 1986, pp. 3-25.

111. J.L. Massey, "An introduction to contemporary cryptology," in "Contemporary Cryptology: The Science of Information Integrity," G.J. Simmons, Ed., IEEE Press, 1991, pp. 3-39.

112. S.M. Matyas, C.H. Meyer, J. Oseas, "Generating strong one-way functions with cryptographic algorithm," IBM Techn. Disclosure Bull., Vol. 27, No. 10A, 1985, pp. 5658-5659.

113. K. Mehlhorn, U. Vishkin, "Randomized and deterministic simulations of PRAMs by parallel machines with restricted granularity of parallel memories," Acta Informatica, Vol. 21, Fasc. 4, 1984, pp. 339-374.

114. A. Menezes, Elliptic Curve Public-Key Cryptosystems, Kluwer Academic Publishers, 1993.

115. A.J. Menezes, P.C. van Oorschot, S.A. Vanstone, Handbook of Applied Cryptography," CRC Press, 1997.

116. R. Merkle, "Secrecy, Authentication, and Public Key Systems," UMI Research Press, 1979.

117. R. Merkle, "A certified digital signature," Advances in Cryptology, Proceedings Crypto'89, LNCS 435, G. Brassard, Ed., Springer-Verlag, 1990, pp. 218-238.

118. R. Merkle, "One way hash functions and DES," Advances in Cryptology, Proceedings Crypto'89, LNCS 435, G. Brassard, Ed., Springer-Verlag, 1990, pp. 428-446.

119. R. Merkle, "A fast software one-way hash function," Journal of Cryptology, Vol. 3, No. 1, 1990, pp. 43-58.

120. R. Merkle, M. Hellman, "Hiding information and signatures in trapdoor knapsacks," IEEE Trans. on Information Theory, Vol. IT-24, No. 5, 1978, pp. 525-530.

121. C.H. Meyer, S.M. Matyas, "Cryptography: a New Dimension in Data Security," Wiley \& Sons, 1982.

122. C.H. Meyer, M. Schilling, "Secure program load with Manipulation Detection Code," Proc. Securicom 1988, pp. 111-130.

Appeared in State of the Art and Evolution of Computer Security and Industrial Cryptography, Lecture Notes in Computer Science 1528, Springer-Verlag, pp. 50-105. (C)1998 Springer-Verlag 
123. C. Mitchell, "Multi-destination secure electronic mail," The Computer Journal, Vol. 32, No. 1, 1989, pp. 13-15.

124. S. Miyaguchi, M. Iwata, K. Ohta, "New 128-bit hash function," Proc. 4th International Joint Workshop on Computer Communications, Tokyo, Japan, July 13-15, 1989, pp. 279-288.

125. S. Miyaguchi, K. Ohta, M. Iwata, "128-bit hash function (N-hash)," Proc. Securicom 1990, pp. 127-137.

126. J.H. Moore, G.J. Simmons, "Cycle structure of the DES for keys having palindromic (or antipalindromic) sequences of round keys," IEEE Trans. on Software Engineering, Vol. 13, 1987, pp. 262-273.

127. J.H. Moore, "Protocol failures in cryptosystems," in "Contemporary Cryptology: The Science of Information Integrity," G.J. Simmons, Ed., IEEE Press, 1991, pp. 543-558.

128. M. Naor, M. Yung, "Universal one-way hash functions and their cryptographic applications," Proc. 21st ACM Symposium on the Theory of Computing, 1990, pp. $387-394$.

129. A.M. Odlyzko, "The rise and fall of knapsack cryptosystems," Cryptology and Computational Number Theory, C. Pomerance, Ed., Proc. Sympos. Appl. Math., Vol. 42, American Mathematical Society, 1990, pp. 75-88.

130. T. Okamoto, "Provably secure and practical identification schemes and corresponding signature schemes," Advances in Cryptology, Proceedings Crypto'92, LNCS 740, E.F. Brickell, Ed., Springer-Verlag, 1993, pp. 31-53.

131. T. Okamoto, K. Ohta, "A modification of the Fiat-Shamir scheme," Advances in Cryptology, Proceedings Crypto'88, LNCS 403, S. Goldwasser, Ed., SpringerVerlag, 1990, pp. 232-243.

132. J. Patarin, "Collisions and inversions for Damgård's whole hash function," $A d$ vances in Cryptology, Proceedings Asiacrypt'94, LNCS 917, J. Pieprzyk and R. Safavi-Naini, Eds., Springer-Verlag, 1995, pp. 307-321.

133. B. Pfitzmann, "Digital Signatures Schemes. General Framework and Fail-Stop Signatures," LNCS 1100, Springer-Verlag, 1996.

134. B. Preneel, "Analysis and design of cryptographic hash functions," Doctoral Dissertation, Katholieke Universiteit Leuven, 1993.

135. B. Preneel, R. Govaerts, J. Vandewalle, "Cryptographically secure hash functions: an overview," ESAT Internal Report, K.U. Leuven, 1989.

136. B. Preneel, R. Govaerts, J. Vandewalle, "On the power of memory in the design of collision resistant hash functions," Advances in Cryptology, Proceedings Auscrypt'92, LNCS 718, J. Seberry and Y. Zheng, Eds., Springer-Verlag, 1993, pp. $105-121$.

137. B. Preneel, R. Govaerts, J. Vandewalle, "Hash functions based on block ciphers: a synthetic approach," Advances in Cryptology, Proceedings Crypto'93, LNCS 773, D. Stinson, Ed., Springer-Verlag, 1994, pp. 368-378.

138. B. Preneel, V. Rijmen, A. Bosselaers, "Recent developments in the design of conventional cryptographic algorithms," This Volume.

139. B. Preneel, V. Rijmen, P.C. van Oorschot, "A security analysis of the Message Authenticator Algorithm (MAA)," European Transactions on Telecommunications, Vol. 8, No. 5, 1997, pp. 455-470.

140. B. Preneel, P.C. van Oorschot, "MDx-MAC and building fast MACs from hash functions," Advances in Cryptology, Proceedings Crypto'95, LNCS 963, D. Coppersmith, Ed., Springer-Verlag, 1995, pp. 1-14.

Appeared in State of the Art and Evolution of Computer Security and Industrial Cryptography, Lecture Notes in Computer Science 1528, Springer-Verlag, pp. 50-105. (C)1998 Springer-Verlag 
141. B. Preneel, P.C. van Oorschot, "On the security of two MAC algorithms," $A d$ vances in Cryptology, Proceedings Eurocrypt'96, LNCS 1070, U. Maurer, Ed., Springer-Verlag, 1996, pp. 19-32.

142. B. Preneel, P.C. van Oorschot, "A key recovery attack on the ANSI X9.19 retail MAC," Electronics Letters, Vol. 32, No. 17, 1996, pp. 1568-1569.

143. J.-J. Quisquater, J.-P. Delescaille, "How easy is collision search ? Application to DES," Advances in Cryptology, Proceedings Eurocrypt'89, LNCS 434, J.J. Quisquater and J. Vandewalle, Eds., Springer-Verlag, 1990, pp. 429-434.

144. J.-J. Quisquater, J.-P. Delescaille, "How easy is collision search. New results and applications to DES," Advances in Cryptology, Proceedings Crypto'89, LNCS 435, G. Brassard, Ed., Springer-Verlag, 1990, pp. 408-413.

145. J.-J. Quisquater, L. Guillou, "A "paradoxical" identity-based signature scheme resulting from zero-knowledge," Advances in Cryptology, Proceedings Crypto'88, LNCS 403, S. Goldwasser, Ed., Springer-Verlag, 1990, pp. 216-231.

146. M.O. Rabin, "Digitalized signatures," in "Foundations of Secure Computation," R. Lipton, R. DeMillo, Eds., Academic Press, New York, 1978, pp. 155-166.

147. M.O. Rabin, "Digitalized signatures and public-key functions as intractable as factorization," Technical Report MIT/LCS/TR-212, Massachusetts Institute of Technology, Laboratory for Computer Science, Cambridge, MA, January 1979.

148. V. Rijmen, B. Preneel, "Improved characteristics for differential cryptanalysis of hash functions based on block ciphers," Fast Software Encryption, LNCS 1008, B. Preneel, Ed., Springer-Verlag, 1995, pp. 242-248.

149. RIPE, "Integrity Primitives for Secure Information Systems. Final Report of RACE Integrity Primitives Evaluation (RIPE-RACE 1040)," LNCS 100\%, A. Bosselaers, B. Preneel, Eds., Springer-Verlag, 1995.

150. R.L. Rivest, "The MD4 message digest algorithm," Advances in Cryptology, Proceedings Crypto'90, LNCS 537, S. Vanstone, Ed., Springer-Verlag, 1991, pp. 303311.

151. R.L. Rivest, "The MD5 message-digest algorithm," Request for Comments (RFC) 1321, Internet Activities Board, Internet Privacy Task Force, April 1992.

152. R.L. Rivest, "All-or-nothing encryption and the package transform," Fast Software Encryption, LNCS 1267, E. Biham, Ed., Springer-Verlag, 1997, pp. 210-218.

153. R.L. Rivest, A. Shamir, L. Adleman, "A method for obtaining digital signatures and public-key cryptosystems," Communications ACM, Vol. 21, February 1978, pp. 120-126.

154. P. Rogaway, "Bucket hashing and its application to fast message authentication," Advances in Cryptology, Proceedings Crypto'95, LNCS 963, D. Coppersmith, Ed., Springer-Verlag, 1995, pp. 29-42.

155. N. Rogier, P. Chauvaud, "MD2 is not secure without the checksum byte," Designs, Codes, and Cryptography, Vol. 12, No. 3, 1997, pp. 245-251.

156. J. Rompel, "One-way functions are necessary and sufficient for secure signatures," Proc. 22nd ACM Symposium on the Theory of Computing, 1990, pp. 387-394.

157. R.A. Rueppel, "Stream ciphers," in "Contemporary Cryptology: The Science of Information Integrity," G.J. Simmons, Ed., IEEE Press, 1991, pp. 65-134.

158. C.P. Schnorr, "Efficient identification and signatures for smart cards," Advances in Cryptology, Proceedings Crypto'89, LNCS 435, G. Brassard, Ed., SpringerVerlag, 1990, pp. 239-252.

159. C.P. Schnorr, S. Vaudenay, "Parallel FFT-Hashing," Fast Software Encryption, LNCS 809, R. Anderson, Ed., Springer-Verlag, 1994, pp. 149-156.

160. C.E. Shannon, "Communication theory of secrecy systems," Bell System Technical Journal, Vol. 28, 1949, pp. 656-715.

Appeared in State of the Art and Evolution of Computer Security and Industrial Cryptography, Lecture Notes in Computer Science 1528, Springer-Verlag, pp. 50-105. (C)1998 Springer-Verlag 
161. V. Shoup, "On fast and provably secure message authentication based on universal hashing, Advances in Cryptology, Proceedings Crypto'96, LNCS 1109, N. Koblitz, Ed., Springer-Verlag, 1996, pp. 313-328.

162. G.J. Simmons, "A survey of information authentication," in "Contemporary Cryptology: The Science of Information Integrity," G.J. Simmons, Ed., IEEE Press, 1991, pp. 381-419.

163. G.J. Simmons, "How to insure that data acquired to verify treat compliance are trustworthy," in "Contemporary Cryptology: The Science of Information Integrity," G.J. Simmons, Ed., IEEE Press, 1991, pp. 615-630.

164. D. Simon, "Finding collisions on a one-way street: Can secure hash functions be based on general assumptions?" Advances in Cryptology, Proceedings Eurocrypt'98, LNCS 1403, K. Nyberg, Ed., Springer-Verlag, 1998, pp. 334-345.

165. D.R. Stinson, "The combinatorics of authentication and secrecy codes," Journal of Cryptology, Vol. 2, No. 1, 1990, pp. 23-49.

166. D.R. Stinson, "Universal hashing and authentication codes," Designs, Codes, and Cryptography, Vol. 4, No. 4, 1994, pp. 369-380. See also Advances in Cryptology, Proceedings Crypto'91, LNCS 576, J. Feigenbaum, Ed., Springer-Verlag, 1992, pp. $74-85$.

167. D.R. Stinson, "Combinatorial characterizations of authentication codes," Designs, Codes, and Cryptography, Vol. 2, No. 2, 1992, pp. 175-187. See also Advances in Cryptology, Proceedings Crypto'91, LNCS 576, J. Feigenbaum, Ed., SpringerVerlag, 1992, pp. 62-73.

168. J.-P. Tillich, G. Zémor, "Hashing with $S L_{2}$," Advances in Cryptology, Proceedings Crypto'94, LNCS 839, Y. Desmedt, Ed., Springer-Verlag, 1994, pp. 40-49.

169. P.C. van Oorschot, M.J. Wiener, "Parallel collision search with application to hash functions and discrete logarithms," Proc. 2nd ACM Conference on Computer and Communications Security, ACM, 1994, pp. 210-218 (final version to appear in Journal of Cryptology).

170. G.S. Vernam, "Cipher printing telegraph system for secret wire and radio telegraph communications," Journal American Institute of Electrical Engineers, Vol. XLV, 1926, pp. 109-115.

171. M.N. Wegman, J.L. Carter, "New hash functions and their use in authentication and set equality," Journal of Computer and System Sciences, Vol. 22, No. 3, 1981, pp. 265-279.

172. A.C. Yao, "Theory and applications of trapdoor functions," Proc. 23rd IEEE Symposium on Foundations of Computer Science, 1982, pp. 80-91.

173. G. Yuval, "How to swindle Rabin," Cryptologia, Vol. 3, 1979, pp. 187-189.

174. G. Zémor, "Hash functions and Cayley graphs," Designs, Codes, and Cryptography, Vol. 4, No. 4, 1994, pp. 381-394.

175. Y. Zheng, T. Matsumoto, H. Imai, "Connections between several versions of oneway hash functions," Proc. SCIS90, The 1990 Symposium on Cryptography and Information Security, Nihondaira, Japan, Jan. 31-Feb. 2, 1990.

176. Y. Zheng, J. Pieprzyk, J. Seberry, "HAVAL - a one-way hashing algorithm with variable length output," Advances in Cryptology, Proceedings Auscrypt'92, LNCS 718, J. Seberry and Y. Zheng, Eds., Springer-Verlag, 1993, pp. 83-104.

Appeared in State of the Art and Evolution of Computer Security and Industrial Cryptography, Lecture Notes in Computer Science 1528, Springer-Verlag, pp. 50-105. (c)1998 Springer-Verlag 